\title{
CONVOLUTION POWERS IN THE OPERATOR-VALUED FRAMEWORK
}

\author{
MICHAEL ANSHELEVICH, SERBAN T. BELINSCHI, MAXIME FEVRIER, \\ AND ALEXANDRU NICA
}

\begin{abstract}
We consider the framework of an operator-valued noncommutative probability space over a unital $C^{*}$-algebra $\mathcal{B}$. We show how for a $\mathcal{B}$-valued distribution $\mu$ one can define convolution powers $\mu^{\boxplus \eta}$ (with respect to free additive convolution) and $\mu^{\uplus \eta}$ (with respect to Boolean convolution), where the exponent $\eta$ is a suitably chosen linear map from $\mathcal{B}$ to $\mathcal{B}$, instead of being a nonnegative real number. More precisely, $\mu^{\uplus \eta}$ is always defined when $\eta$ is completely positive, while $\mu^{\boxplus \eta}$ is always defined when $\eta-1$ is completely positive (with "1" denoting the identity map on $\mathcal{B}$ ).

In connection to these convolution powers we define an evolution semigroup $\left\{\mathbb{B}_{\eta} \mid \eta: \mathcal{B} \rightarrow \mathcal{B}\right.$, completely positive $\}$, related to the Boolean BercoviciPata bijection. We prove several properties of this semigroup, including its connection to the $\mathcal{B}$-valued free Brownian motion.

We also obtain two results on the operator-valued analytic function theory related to convolution powers $\mu^{\boxplus \eta}$. One of the results concerns the analytic subordination of the Cauchy-Stieltjes transform of $\mu^{\boxplus \eta}$ with respect to the Cauchy-Stieltjes transform of $\mu$. The other one gives a $\mathcal{B}$-valued version of the inviscid Burgers equation, which is satisfied by the Cauchy-Stieltjes transform of a $\mathcal{B}$-valued free Brownian motion.
\end{abstract}

\section{INTRODUCTION}

1.1. Convolution powers with respect to $\boxplus$. The study of free probability was initiated by Voiculescu in the early 1980s, and combinatorial methods were introduced to it by Speicher in the early 1990s. Soon thereafter both the analytic and the combinatorial aspects of the theory were extended (in [20] and respectively [16]) to an operator-valued framework. Very roughly, the operator-valued framework is analogous to conditional probability: instead of working with an expectation functional (for noncommutative random variables) which takes values in $\mathbb{C}$, one works with a conditional expectation taking values in an algebra $\mathcal{B}$. The $\mathcal{B}$-valued framework adds further depth to the theory; a notable example of this appears for instance in the relation between free probability and random matrices, where the paper of Shlyakhtenko [14] found relations between operator-valued free probability and random band matrices.

Received by the editors August 6, 2011.

2010 Mathematics Subject Classification. Primary 46L54.

The first author was supported in part by NSF grant DMS-0900935.

The second author was supported in part by a Discovery Grant from NSERC, Canada, and by a University of Saskatchewan start-up grant.

The third author was supported in part by grant ANR-08-BLAN-0311-03 from Agence Nationale de la Recherche, France.

The fourth author was supported in part by a Discovery Grant from NSERC, Canada. 
An important role in free probability is played by the free additive convolution $\boxplus$. This is an operation on distributions which reflects the operation of addition for free random variables. When considered in connection to bounded selfadjoint variables in a $\mathbb{C}$-valued framework, $\boxplus$ is an operation on compactly supported probability measures on $\mathbb{R}$, and was studied from the very beginning of the theory [18, 19]. Also from the very beginning, Voiculescu 19 introduced the concept of an $R$-transform $R_{\mu}$ for such a probability measure $\mu$, and proved the linearization property that $R_{\mu \boxplus \nu}=R_{\mu}+R_{\nu}$. By using the $R$-transform one can moreover introduce convolution powers with respect to $\boxplus$ : for a compactly supported probability measure $\mu$ and a real number $t \geq 0$, the convolution power $\mu^{\boxplus t}$ (when it exists) is the probability measure determined uniquely by the fact that

$$
R_{\mu^{\boxplus t}}=t \cdot R_{\mu} .
$$

It is known [1] that $\mu^{\boxplus t}$ is always defined when $t \geq 1$. On the other hand there exist special distributions (the so-called infinitely divisible ones) where the " $\boxplus t$ " powers are defined for every $t \geq 0$. An important such example is provided by the standard semicircular distribution $\gamma$, which is the analog in free probability for the normal law. If we denote by $\gamma_{t}$ the centered semicircular distribution with variance $t$ (so that $\gamma$ becomes $\gamma_{1}$ ), then, in full analogy to the heat semigroup, these form a semigroup: $\gamma_{t} \boxplus \gamma_{s}=\gamma_{t+s}$ for every $s, t \geq 0$. In other words, one has that

$$
\gamma_{t}=\gamma^{\boxplus t}, \quad \forall t \geq 0 .
$$

Now let us move to an operator-valued framework. Throughout the paper, $\mathcal{B}$ will be a fixed unital $C^{*}$-algebra. The $\mathcal{B}$-valued semicircular distributions form a class of examples that are relatively well understood (see e.g. [15]). Here the centered semicircular distributions are again indexed by their variances, but now these variances are allowed to be arbitrary completely positive maps $\eta: \mathcal{B} \rightarrow \mathcal{B}(!)$ This motivates a question, explicitly asked by Hari Bercovici, of defining convolution powers $\mu^{\boxplus \eta}$ for general $\mathcal{B}$-valued distributions $\mu$, so that in particular one obtains the $\mathcal{B}$-valued analogue of (1.2):

$$
\gamma_{\eta}=\gamma^{\boxplus \eta}
$$

where " $\gamma$ " now stands for the centered semicircular distribution having variance equal to the identity map on $\mathcal{B}$.

The answer to this question is one of the main results of this paper, Theorem 7.9 . Since the rescaling by $t$ from (1.1) can be viewed as a particular case of composing the $R$-transform with a positive map from $\mathcal{B}$ to $\mathcal{B}$, we consider the problem of defining convolution powers $\mu^{\boxplus \eta}$ via the formula

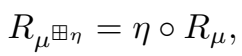

where $\eta: \mathcal{B} \rightarrow \mathcal{B}$ is a completely positive map. Theorem 7.9 says that (1.4) meaningfully defines a distribution $\mu^{\boxplus \eta}$ whenever $\eta: \mathcal{B} \rightarrow \mathcal{B}$ is such that $\eta-1$ is a completely positive map (and where "1" stands for the identity map on $\mathcal{B}$ ).

Here is the moment to make a clarification of our notation. We will use the notation $\Sigma(\mathcal{B})$ for the space of all $\mathcal{B}$-valued distributions; the elements of $\Sigma(\mathcal{B})$ are thus positive $\mathcal{B}$-bimodule maps $\mu: \mathcal{B}\langle\mathcal{X}\rangle \rightarrow \mathcal{B}$ (see Section 2 below for notational details). A smaller class $\Sigma^{0}(\mathcal{B})$ corresponds to the compactly supported distributions from the $\mathbb{C}$-valued case. On the other hand we will use the notation $\Sigma_{\text {alg }}(\mathcal{B})$ for the larger space of all unital $\mathcal{B}$-bimodule maps $\mu: \mathcal{B}\langle\mathcal{X}\rangle \rightarrow \mathcal{B}$. It is easily seen 
that (1.4) can be invoked to define the convolution power $\mu^{\boxplus \eta} \in \Sigma_{\text {alg }}(\mathcal{B})$ for every $\mu \in \Sigma_{\text {alg }}(\mathcal{B})$ and every linear map $\eta: \mathcal{B} \rightarrow \mathcal{B}$. The point of Theorem 7.9 is that upon starting with $\mu$ in the smaller space $\Sigma(\mathcal{B})$ and with $\eta$ as described above, the resulting convolution power $\mu^{\boxplus \eta}$ still belongs to $\Sigma(\mathcal{B})$. In order to arrive at this point, a key role in our considerations will be played by the interaction between free probability and a simpler form of noncommutative probability, called Boolean probability. We elaborate on this interaction in the next subsection.

1.2. Relations with Boolean probability. Boolean probability has an operation of Boolean convolution $\uplus$, which reflects the operation of addition for Boolean independent random variables. This in turn has a linearizing transform, which will be denoted in this paper by $B_{\mu}$. (Usually the linearizing transform for $\uplus$ is rather denoted as " $\eta_{\mu}$ ", but in this paper $\eta$ is reserved for denoting variance linear maps on $\mathcal{B}$.) If $\mu$ is a compactly supported probability measure on $\mathbb{R}$, then one defines convolution powers with respect to $\uplus$ by using the suitable rescaling of the $B$-transform,

$$
B_{\mu^{\uplus t}}=t \cdot B_{\mu} .
$$

So far this parallels very closely the development of $\boxplus$-powers, with $B_{\mu}$ being the Boolean counterpart of $R_{\mu}$. However, due to the simpler nature of Boolean probability, one now has [17] that $\mu^{\uplus t}$ is defined for every compactly supported probability measure on $\mathbb{R}$ and every $t \geq 0$. (In the Boolean world, every $\mu$ is $\uplus$-infinitely divisible.) This fortunate fact can then be put to use in free probability due to the existence of a special bijection, called the Boolean Bercovici-Pata bijection [8, which links the $R$-transform to the $B$-transform. Moreover, the Boolean Bercovici-Pata bijection can be incorporated $\left[3\right.$ into a semigroup $\left\{\mathbb{B}_{t} \mid t \geq 0\right\}$ of transformations on probability measures, which are explicitly defined in terms of convolution powers:

$$
\mathbb{B}_{t}(\mu):=\left(\mu^{\boxplus(1+t)}\right)^{\uplus(1+t)^{-1}},
$$

holding for every probability measure $\mu$ on $\mathbb{R}$ and every $t \geq 0$. The original Boolean Bercovici-Pata bijection is $\mathbb{B}=\mathbb{B}_{1}$. The transformations $\mathbb{B}_{t}$ are sometimes said to give an "evolution towards $\boxplus$-infinite divisibility", due to the fact that $\mathbb{B}_{t}(\mu)$ is $\boxplus$-infinite divisible for every $\mu$ and whenever $t \geq 1$.

The considerations from the preceding paragraph were given in the $\mathbb{C}$-valued framework. But the interactions between free and Boolean probability turn out to continue to hold when one goes to an operator-valued framework. Let us first concentrate on the sheer algebraic and combinatorial aspects of how this happens. Paralleling the $\boxplus$ case, it is easily seen that one can define the convolution power $\mu^{\uplus \eta} \in \Sigma_{\text {alg }}(\mathcal{B})$ for every $\mu \in \Sigma_{\text {alg }}(\mathcal{B})$ and every linear map $\eta: \mathcal{B} \rightarrow \mathcal{B}$, by simply making the requirement that

$$
B_{\mu}^{\uplus \eta}=\eta \circ B_{\mu} .
$$

It is however nontrivial how to combine the generalized convolution powers from (1.4) and (1.7) in order to create, for a general linear map $\alpha: \mathcal{B} \rightarrow \mathcal{B}$, a transformation $\mathbb{B}_{\alpha}$ on $\Sigma_{\text {alg }}(\mathcal{B})$ which is the analogue of $\mathbb{B}_{t}$ from (1.6). This requires some departure from the techniques used previously in the $\mathbb{C}$-valued case, and is achieved in Section 6 of the paper. In Theorem 6.4 we prove that the transformations $\mathbb{B}_{\alpha}: \Sigma_{\text {alg }}(\mathcal{B}) \rightarrow \Sigma_{\text {alg }}(\mathcal{B})$ which are obtained form a commutative (!) 
semigroup:

$$
\mathbb{B}_{\alpha} \circ \mathbb{B}_{\beta}=\mathbb{B}_{\alpha+\beta}, \quad \forall \alpha, \beta: \mathcal{B} \rightarrow \mathcal{B}
$$

(we emphasize that in (1.8) the linear maps $\alpha$ and $\beta$ are not required to commute). We show moreover that one has the formula

$$
\left(\mathbb{B}_{\alpha}(\mu)\right)^{\uplus(1+\alpha)}=\mu^{\boxplus(1+\alpha)},
$$

holding for every $\mu \in \Sigma_{a l g}(\mathcal{B})$ and every linear map $\alpha: \mathcal{B} \rightarrow \mathcal{B}$; so in the special case when $1+\alpha$ is invertible, one can raise both sides of (1.9) to the power $\uplus(1+\alpha)^{-1}$ in order to obtain a faithful analog of the formula (1.6) from the $\mathbb{C}$-valued framework.

While the space $\Sigma_{\text {alg }}(\mathcal{B})$ provides a nice larger environment which is good for algebraic manipulations, our interest really lies in the smaller spaces $\Sigma(\mathcal{B})$ and $\Sigma^{0}(\mathcal{B})$, consisting of distributions which can appear in the $C^{*}$-framework. Thus it is of certain interest to look at the case when the linear maps $\alpha, \beta, \eta$ that were considered above are completely positive (on the unital $C^{*}$-algebra $\mathcal{B}$ that is fixed throughout the paper), and to establish conditions under which the corresponding convolution powers and/or transformations $\mathbb{B}_{\alpha}$ leave $\Sigma(\mathcal{B})$ and $\Sigma^{0}(\mathcal{B})$ invariant. We obtain this by using some suitable operator models for distributions in $\Sigma(\mathcal{B})$. Two such (relatively simple) operator models are described in Section 7 of the paper, and are used to prove that:

(i) $\mu^{\uplus \eta} \in \Sigma(\mathcal{B})$ whenever $\mu \in \Sigma(\mathcal{B})$ and $\eta: \mathcal{B} \rightarrow \mathcal{B}$ is a completely positive linear map (cf. Theorem 7.5);

(ii) $\mathbb{B}_{\alpha}(\mu) \in \Sigma(\mathcal{B})$ whenever $\mu \in \Sigma(\mathcal{B})$ and $\alpha: \mathcal{B} \rightarrow \mathcal{B}$ is a completely positive linear map (cf. Theorem 7.8).

Finally, we can now return to the point left at the end of subsection 1.1 , and explain why is it that $\mu^{\boxplus \eta} \in \Sigma(\mathcal{B})$ whenever $\mu \in \Sigma(\mathcal{B})$ and $\eta: \mathcal{B} \rightarrow \mathcal{B}$ is such that $\eta-1$ is completely positive: denoting $\eta-1=: \alpha$, we see from (1.9) that the distribution $\mu^{\boxplus \eta}$ (which a priori lives in the larger space $\Sigma_{a l g}(\mathcal{B})$ ) satisfies

$$
\mu^{\boxplus \eta}=\left(\mathbb{B}_{\alpha}(\mu)\right)^{\uplus \eta},
$$

but the distribution on the right-hand side of the latter equation does belong to $\Sigma(\mathcal{B})$, by virtue of the results (i) and (ii) indicated above.

1.3. Relations to free Brownian motion, and to analytic functions. Consider again the $\mathbb{C}$-valued framework, and the semigroup of semicircular distributions $\left\{\gamma_{t} \mid t \geq 0\right\}$ from (1.2). For a probability measure $\mu$ on $\mathbb{R}$, the process $\left\{\mu \boxplus \gamma_{t} \mid t \geq 0\right\}$ is called the free Brownian motion started at $\mu$. In [3, 4] it was observed that the transformations $\mathbb{B}_{t}$ from (1.6) are related to the free Brownian motion via an evolution equation of the form

$$
\mathbb{B}_{t}(\Phi(\mu))=\Phi\left(\mu \boxplus \gamma_{t}\right), \quad t \geq 0,
$$

where $\Phi$ is a special transformation on probability measures (not depending on $\mu$ or $t$ ). In this paper we introduce the $\mathcal{B}$-valued analog of the transformation $\Phi$ (defined on lines similar to those of [4]), and we obtain the corresponding evolution equation, where the role of the time parameter is now taken by a linear map $\eta: \mathcal{B} \rightarrow \mathcal{B}$. That is, we have that

$$
\mathbb{B}_{\eta}[\Phi[\mu]]=\Phi\left[\mu \boxplus \gamma_{\eta}\right]
$$


holding for $\mu \in \Sigma(\mathcal{B})$ and $\eta: \mathcal{B} \rightarrow \mathcal{B}$ completely positive. This is obtained in Theorem 6.9 (in a plain algebraic version) and in Corollary 7.11 (in the completely positive version).

In a related development, Section 8 of the paper establishes some results concerning the operator-valued analytic function theory related to the convolution powers $\mu^{\boxplus \eta}$.

On the one hand we show that for $\eta$ as in Theorem 7.9 (that is, an $\eta$ such that $\eta-1$ is completely positive) one has analytic subordination of the Cauchy-Stieltjes transform of $\mu^{\boxplus \eta}$ with respect to the Cauchy-Stieltjes transform of $\mu$. This is the $\mathcal{B}$-valued analogue of a known fact from the $\mathbb{C}$-valued framework [5, 6, but where now the subordination function is an analytic self-map of the set $\{b \in \mathcal{B} \mid \Im b>0\}$.

On the other hand, we show that the Cauchy-Stieltjes transform of the $\mathcal{B}$-valued free Brownian motion started at a distribution $\mu \in \Sigma^{(0)}(\mathcal{B})$ satisfies a $\mathcal{B}$-valued version of the inviscid Burgers equation. The occurrence of the Burgers equation in free probability came with a fundamental result of Voiculescu, where (in the $\mathbb{C}$ valued framework) the complex Burgers equation was found to be the free analogue of the heat equation. This means, more precisely, that the complex Burgers equation with initial data the Cauchy-Stieltjes transform of a given probability measure $\mu$ on $\mathbb{R}$ is solved by the Cauchy-Stieltjes transform of the free Brownian motion started at $\mu$. Theorem 8.3 of the present paper establishes the $\mathcal{B}$-valued analog of this (in fact of a slightly stronger result, given in [3], which is expressed in terms of the transformations $\mathbb{B}_{t}$ ).

1.4. Organization of the paper. Besides the present introduction, the paper has eight other sections. Section 2 reviews some general background from $\mathcal{B}$-valued noncommutative probability; then in Section 3 we set up the $\mathcal{B}$-series machinery and the nesting structures corresponding to noncrossing partitions. Sections 4 and 5 set up basic definitions and results concerning the operator-valued $R$ and $B$-transforms, and concerning a transformation $\widehat{\mathrm{RB}}$ (defined on $\mathcal{B}$-series) which connects them. The definition of convolution powers $\mu^{\boxplus \eta}$ and $\mu^{\uplus \eta}$ is also given here. In Section 6, the transformations $\mathbb{B}_{\eta}$ are defined and shown to form a semigroup, and the evolution equation is proved. In Section 7 we describe the operator models and use them to prove the positivity results mentioned in subsection 1.2 above. Section 8 contains the results on operator-valued analytic function theory which were announced in subsection 1.3. Finally, the main result in Section 9 is an alternative operator model for Boolean convolution powers.

\section{Preliminaries}

2.1. Free $\mathcal{B}$-bimodule. Throughout the paper $\mathcal{B}$ will be a $C^{*}$-algebra. For $\mathcal{X}$ a formal variable, denote by

$$
\mathcal{B}\langle\mathcal{X}\rangle=\mathcal{B} \oplus \mathcal{B} \mathcal{X} \mathcal{B} \oplus \mathcal{B X} \mathcal{B X} \mathcal{B} \oplus
$$

the algebra of all polynomials in $\mathcal{X}$, with coefficients in $\mathcal{B}$, and by

$$
\mathcal{B}_{0}\langle\mathcal{X}\rangle=\mathcal{B} \mathcal{X} \mathcal{B} \oplus \mathcal{B X} \mathcal{B} \mathcal{X} \mathcal{B} \oplus
$$


the polynomials without constant term. We will assume that $\mathcal{X}$ and $\mathcal{B}$ are algebraically independent. This means (by definition) that (2.1) amounts to

$$
\mathcal{B}\langle\mathcal{X}\rangle \simeq \bigoplus_{n=1}^{\infty} \mathcal{B}^{\otimes_{\mathbb{C}}^{n}}
$$

the tensor product being over $\mathbb{C}$. The set $\mathcal{B}\langle\mathcal{X}\rangle$ is a $\mathcal{B}$-bimodule in the obvious way.

In particular, a collection of $\mathbb{C}$-linear maps $\mu_{n}: \mathcal{B}^{\otimes \mathbb{C}(n-1)} \rightarrow \mathcal{B}$ can be combined via

$$
\mu\left[\mathcal{X} b_{1} \mathcal{X} b_{2} \cdots b_{n-1} \mathcal{X}\right]=\mu_{n}\left(b_{1} \otimes b_{2} \otimes \ldots \otimes b_{n-1}\right)
$$

into a $\mathcal{B}$-bimodule map

$$
\mu: \mathcal{B}\langle\mathcal{X}\rangle \rightarrow \mathcal{B}
$$

such that $\mu[b]=b$ for $b \in \mathcal{B}$. As already mentioned in the introduction, the set of all such maps will be denoted by $\Sigma_{\text {alg }}(\mathcal{B})$.

If such a $\mu$ is positive, we will refer to it as a conditional expectation and omit the term "positive". Since $\mathcal{B}$ is a $C^{*}$-algebra, by Proposition 3.5.4 of [16, in this case $\mu$ is automatically completely positive. We will denote

$$
\Sigma(\mathcal{B})=\{(\text { positive }) \text { conditional expectations } \mu: \mathcal{B}\langle\mathcal{X}\rangle \rightarrow \mathcal{B}\} .
$$

\section{2. $\mathcal{B}$-series.}

Definition 2.1. $1^{o}$ We will use the name $\mathcal{B}$-series for objects of the form

$$
F=\left(\beta_{n}\right)_{n \geq 1},
$$

where, for every $n \geq 1, \beta_{n}: \mathcal{B}^{n-1} \rightarrow \mathcal{B}$ is a $\mathbb{C}$-multilinear functional (with the convention that $\beta_{1}$ is an element of $\left.\mathcal{B}\right)$. The set of all $\mathcal{B}$-series will be denoted by $\operatorname{Ser}(\mathcal{B})$.

$2^{o}$ For a series $F$ as in (2.2), the functionals $\beta_{n}$ will be referred to as terms of $F$. We will use the notation

$$
F^{[n]}:=(n \text {-th term of } F), \quad \text { for } F \in \operatorname{Ser}(\mathcal{B}) \text { and } n \geq 1 .
$$

$3^{\circ}$ On $\operatorname{Ser}(\mathcal{B})$ we have some natural operations. In the present paper it is particularly important that for every $\mathbb{C}$-linear map $\alpha: \mathcal{B} \rightarrow \mathcal{B}$ and every series $F \in \operatorname{Ser}(\mathcal{B})$ one can define the composition $\alpha \circ F \in \operatorname{Ser}(\mathcal{B})$ by putting

$$
(\alpha \circ F)^{[n]}:=\alpha \circ F^{[n]}, \quad \forall n \geq 1
$$

(for $n=1$, this just means that $\left.(\alpha \circ F)^{[1]}:=\alpha\left(F^{[1]}\right) \in \mathcal{B}\right)$.

It is also clear that for $F_{1}, F_{2} \in \operatorname{Ser}(\mathcal{B})$ and $\lambda_{1}, \lambda_{2} \in \mathbb{C}$ one can define the linear combination $\lambda_{1} F_{1}+\lambda_{2} F_{2} \in \operatorname{Ser}(\mathcal{B})$ by putting

$$
\left(\lambda_{1} F_{1}+\lambda_{2} F_{2}\right)^{[n]}:=\lambda_{1} \cdot F_{1}^{[n]}+\lambda_{2} \cdot F_{2}^{[n]}, \quad \forall n \geq 1 .
$$

This extends naturally to a $\mathcal{B}$-bimodule structure, where for $b \in \mathcal{B}$ and $F \in \operatorname{Ser}(\mathcal{B})$ the new series $b F$ and $F b$ are obtained by taking the linear map $\alpha$ from (2.4) to be given by left (respectively right) multiplication with $b$ on $\mathcal{B}$.

Definition 2.2. Let $\mu$ be a distribution in $\Sigma_{a l g}(\mathcal{B})$. The moment series of $\mu$ is the series $M_{\mu} \in \operatorname{Ser}(\mathcal{B})$ with terms defined as follows: $M_{\mu}^{[1]}=\mu(\mathcal{X})$ and

$$
M_{\mu}^{[n]}\left(b_{1}, \ldots, b_{n-1}\right)=\mu\left[\mathcal{X} b_{1} \mathcal{X} b_{2} \ldots \mathcal{X} b_{n-1} \mathcal{X}\right]
$$

for every $n \geq 2$ and $b_{1}, \ldots, b_{n-1} \in \mathcal{B}$. 
Remark 2.3. Clearly, the correspondence $\mu \mapsto M_{\mu}$ is a bijection between $\Sigma_{\text {alg }}(\mathcal{B})$ and $\operatorname{Ser}(\mathcal{B})$. The example of moment series also explains why in Definition 2.1 we used the notation $F^{[n]}$ for a function of $n-1$ arguments (the functional $M_{\mu}^{[n]}$ : $\mathcal{B}^{n-1} \rightarrow \mathcal{B}$ really is some kind of "moment of order $n$ " for $\mu$ ).

Remark 2.4. Let $F$ be a $\mathcal{B}$-series and let $b$ be an element of $\mathcal{B}$. In preparation for analytic considerations that will show up later in the paper, we mention that we will use the notation

$$
F(b):=F^{[1]}+\sum_{n=2}^{\infty} F^{[n]}(b, \ldots, b) \in \mathcal{B}
$$

whenever the sum on the right-hand side of this equality converges (in the norm topology of the $C^{*}$-algebra $\mathcal{B}$ ).

Remark 2.5. In order to justify the terminology introduced above, let us look for a moment at what this means in the special case when $\mathcal{B}=\mathbb{C}$. In this case, what one does is to take a series with complex coefficients

$$
f(z)=\sum_{n=1}^{\infty} \alpha_{n} z^{n-1}
$$

and convert it into $F=\left(F^{[n]}\right)_{n \geq 1}$, with $F^{[1]}=\alpha_{1} \in \mathbb{C}$ and where $F^{[n]}: \mathbb{C}^{n-1} \rightarrow \mathbb{C}$ is defined by

$$
F^{[n]}\left(z_{1}, \ldots, z_{n-1}\right)=\alpha_{n} z_{1} \cdots z_{n-1}, \quad \forall n \geq 2 \text { and } z_{1}, \ldots, z_{n-1} \in \mathbb{C} .
$$

So here every $F^{[n]}$ is indeed a "term" in the writing of the series $f(z)$ (under suitable convergence hypotheses, the right-hand side of (2.7) is the infinite sum $\left.\sum_{n=1}^{\infty} F^{[n]}(z, z, \ldots, z)\right)$.

2.3. Distributions in a $\mathcal{B}$-valued $C^{*}$-probability space. A $\mathcal{B}$-valued $C^{*}$-probability space is a pair $(\mathcal{M}, \mathbb{E})$ where $\mathcal{M}$ is a $C^{*}$-algebra such that $\mathcal{M} \supseteq \mathcal{B}$ and $\mathbb{E}: \mathcal{M} \rightarrow \mathcal{B}$ is a conditional expectation (a unital positive $\mathcal{B}$-bimodule map).

If $(\mathcal{M}, \mathbb{E})$ is a $\mathcal{B}$-valued $C^{*}$-probability space and if $X=X^{*} \in \mathcal{M}$, then one defines the distribution of $X$ as the conditional expectation $\mu_{X} \in \Sigma(\mathcal{B})$ given by

$$
\mu_{X}\left[b_{0} \mathcal{X} b_{1} \mathcal{X} \cdots \mathcal{X} b_{n}\right]=\mathbb{E}\left[b_{0} X b_{1} X \cdots X b_{n}\right] .
$$

We will denote by

$$
\Sigma^{0}(\mathcal{B}) \subset \Sigma(\mathcal{B})
$$

the set of all $\mu$ arising in this way. Equivalently, $\mu \in \Sigma^{0}(\mathcal{B})$ if for any state $\phi$ on $\mathcal{B}$, the operator $\mathcal{X}$ in the GNS representation of $(\mathcal{B}\langle\mathcal{X}\rangle, \phi \circ \mu)$ is bounded. More explicitly this is the case if for some $M>0$ and all $b_{1}, b_{2}, \ldots, b_{n-1} \in \mathcal{B}$,

$$
\left\|\mu\left[\mathcal{X} b_{1} \mathcal{X} \ldots b_{n-1} \mathcal{X}\right]\right\| \leq M^{n}\left\|b_{1}\right\| \cdot\left\|b_{2}\right\| \cdot \ldots \cdot\left\|b_{n-1}\right\| .
$$

Moreover, the moment series of $X$ is defined to be

$$
M_{X}:=M_{\mu_{X}} \in \operatorname{Ser}(\mathcal{B}) .
$$

Or in other words, one has $M_{X}^{[1]}=\mathbb{E}[X]$ and

$$
M_{X}^{[n]}\left(b_{1}, \ldots, b_{n-1}\right)=\mathbb{E}\left[X b_{1} X b_{2} \cdots X b_{n-1} X\right]
$$


for every $n \geq 2$ and $b_{1}, \ldots, b_{n-1} \in \mathcal{B}$. It is worth noting that

$$
M_{X}(b)=\sum_{n=1}^{\infty} M_{X}^{[n]}(b, \ldots, b)
$$

is an analytic map on $\left\{b \in \mathcal{B}:\|b\|<\|X\|^{-1}\right\}$.

We also mention here that the generalized resolvent (or operator-valued CauchyStieltjes transform) of the distribution $\mu_{X}$ of a random variable $X$ is defined by

$$
G_{\mu_{X}}(b)=\mathbb{E}\left[(b-X)^{-1}\right] .
$$

This function of $b$ is analytic on the set of elements $b \in \mathcal{B}$ for which $b-X$ is invertible. The Cauchy-Stieltjes transform is particularly relevant in the context of fully matricial sets and maps 21]. Its natural domain in the $C^{*}$-algebraic context is the set $\{b \in \mathcal{B}: \Im b>0\}$. (By $\Im b>0$ we mean that there exists some $\varepsilon>0$ so that $\Im b=\left(b-b^{*}\right) /(2 i) \geq \varepsilon \cdot 1$.) However, the equality

$$
G_{\mu_{X}}(b)=b^{-1}\left(M_{X}\left(b^{-1}\right)+1\right) b^{-1}
$$

is easily seen to be true for $b$ invertible with $\left\|b^{-1}\right\|<\|X\|^{-1}$.

2.4. Independence and convolution. Let $(\mathcal{M}, \mathbb{E})$ be a $\mathcal{B}$-valued $C^{*}$-probability space, and let $\mathcal{M}_{1}, \mathcal{M}_{2}, \ldots, \mathcal{M}_{k}$ be subalgebras of $\mathcal{M}$ which contain $\mathcal{B}$. These subalgebras are said to be freely independent with respect to $\mathbb{E}$ when the following happens: for any $j(i) \neq j(i+1), X_{i} \in \mathcal{M}_{j(i)}$ with $\mathbb{E}\left[X_{i}\right]=0$, and for any $b_{0}, \ldots, b_{n} \in$ $\mathcal{B}$, we have

$$
\mathbb{E}\left[b_{0} X_{1} b_{2} X_{2} \ldots X_{n} b_{n}\right]=0 .
$$

Operators are freely independent if the $*$-subalgebras they generate over $\mathcal{B}$ are freely independent.

Let $(\mathcal{M}, \mathbb{E})$ be a $\mathcal{B}$-valued $C^{*}$-probability space, and suppose we have a decomposition $\mathcal{M}=\mathcal{B} \oplus \mathcal{M}_{0}$ (with multiplication $\left(b_{1}, m_{1}\right) \cdot\left(b_{2}, m_{2}\right)=\left(b_{1} b_{2}, b_{1} m_{2}+m_{1} b_{2}+\right.$ $\left.m_{1} m_{2}\right)$ ). Subalgebras $\mathcal{M}_{1}, \mathcal{M}_{2}, \ldots, \mathcal{M}_{k} \subset \mathcal{M}_{0}$ are Boolean independent with respect to $\mathbb{E}$ if for any $j(i) \neq j(i+1), X_{i} \in \mathcal{M}_{j(i)}$ for $i \neq 1, n, X_{i} \in \mathcal{B} \oplus \mathcal{M}_{j(i)}$, $i=1, n$, and any $b_{0}, \ldots, b_{n} \in \mathcal{B}$ we have

$$
\mathbb{E}\left[b_{0} X_{1} b_{2} X_{2} \ldots X_{n} b_{n}\right]=b_{0} \mathbb{E}\left[X_{1}\right] b_{1} \mathbb{E}\left[X_{2}\right] \ldots \mathbb{E}\left[X_{n}\right] b_{n} .
$$

Operators in $\mathcal{M}_{0}$ are Boolean independent if the $*$-subalgebras of $\mathcal{M}_{0}$ they generate over $\mathcal{B}$ are Boolean independent.

If $\mu, \nu \in \Sigma(\mathcal{B})$, there exist freely independent symmetric (possibly unbounded) operators $X, Y$ with $\mu_{X}=\mu, \mu_{Y}=\nu$. The distribution of $X+Y$ is uniquely determined by $\mu$ and $\nu$, and is their free convolution:

$$
\mu \boxplus \nu:=\mu_{X+Y} \in \Sigma(\mathcal{B}) .
$$

Similarly, if $X$ and $Y$ are chosen to be Boolean independent, their distribution is the Boolean convolution of $\mu$ and $\nu$,

$$
\mu \uplus \nu:=\mu_{X+Y} \in \Sigma(\mathcal{B}) .
$$

As mentioned in the introduction, both $\boxplus$ and $\uplus$ have linearizing transforms, the $R$-transform and respectively the $B$-transform. For $\mu \in \Sigma(\mathcal{B})$ (and more generally, for $\left.\mu \in \Sigma_{\text {alg }}(\mathcal{B})\right)$ the $R$-transform $R_{\mu}$ and the $B$-transform $B_{\mu}$ are series in $\operatorname{Ser}(\mathcal{B})$; their precise definitions will be reviewed in Section 4 below. The linearization property is that

$$
R_{\mu \boxplus \nu}=R_{\mu}+R_{\nu} \text { and respectively } B_{\mu \uplus \nu}=B_{\mu}+B_{\nu} \text {, for every } \mu, \nu \in \Sigma(\mathcal{B}) .
$$




\section{B-SERIES AND NONCROSSING PARTITIONS}

Remark 3.1 ( $N C(n)$ terminology). The workhorse for combinatorial considerations in free probability is the set $N C(n)$ of noncrossing partitions of $\{1, \ldots, n\}$. In connection to it we will use the standard notation and terminology, as appearing for instance in Lecture 9 of the monograph [12. In particular the partitions in $N C(n)$ will be denoted by letters such as $\pi, \rho, \ldots$ (typical notation will be $\pi=$ $\left\{V_{1}, \ldots, V_{k}\right\} \in N C(n)$, where the $V_{i}$ are the blocks of $\left.\pi\right)$. We will also use the customary partial order given on $N C(n)$ by reverse refinement: for $\pi, \rho \in N C(n)$ we write " $\pi \leq \rho$ " to mean that every block of $\rho$ is a union of blocks of $\pi$. The minimal and maximal elements of $(N C(n), \leq)$ are denoted by $0_{n}$ (the partition of $\{1, \ldots, n\}$ into $n$ singleton blocks) and respectively $1_{n}$ (the partition of $\{1, \ldots, n\}$ into only one block).

Remark 3.2 (Nested terms $F^{[\pi]}$ for $F \in \operatorname{Ser}(\mathcal{B})$ and $\pi \in N C(n)$ ). Let a series $F \in \operatorname{Ser}(\mathcal{B})$ be given. In this remark and in the next definition we explain how one naturally constructs a family of $\mathbb{C}$-multilinear functionals $F^{[\pi]}: \mathcal{B}^{n-1} \rightarrow \mathcal{B}$, one such functional for every $n \geq 1$ and every $\pi \in N C(n)$. If $\pi$ happens to be $1_{n}$, then we will just get $F^{\left[1_{n}\right]}=F^{[n]}$, the $n$-th term of the series $F$. For a general $\pi \in N C(n)$, the point of view that works best when defining $F^{[\pi]}$ is to treat $\pi$ as a "recipe for nesting intervals inside each other". Indeed, the idea of nesting intervals has a correspondent in the framework of multilinear functionals, where such functionals are nested inside each other by using parentheses. (Thus if $\pi$ is written explicitly, $\pi=\left\{V_{1}, \ldots, V_{k}\right\}$, then $F^{[\pi]}$ will be obtained by suitably nesting inside each other the functionals $F^{\left[\left|V_{1}\right|\right]}, \ldots, F^{\left[\left|V_{k}\right|\right]}$.) This very fundamental relation between noncrossing partitions and multilinear functionals arising in $\mathcal{B}$ valued noncommutative probability was put into evidence in [16].

We first explain how things go by a concrete example. Consider a functional such as $L: \mathcal{B}^{4} \rightarrow \mathcal{B}$, where

$$
L\left(b_{1}, \ldots, b_{4}\right):=F^{[3]}\left(b_{1} F^{[2]}\left(b_{2}\right) b_{3}, b_{4}\right), \quad \text { for } b_{1}, \ldots, b_{4} \in \mathcal{B} .
$$

In a very "literal" sense (from the point of view of a typesetter) the right-hand side of the above formula is of the form

$$
W_{1} b_{1} W_{2} b_{2} W_{3} b_{3} W_{4} b_{4} W_{5},
$$

where each of $W_{1}, \ldots, W_{5}$ is a string of symbols made up of left and right parentheses, commas, and the occasional " $F^{[m]}$ ". More precisely, we have

$$
\left\{\begin{array}{l}
W_{1}=" F^{[3]}(" \\
W_{2}=" F^{[2]}(" \\
\left.W_{3}="\right) " \text { (only a right bracket) } \\
W_{4}=", " \text { (only a comma) } \\
\left.W_{5}="\right) " \text { (only a right bracket) }
\end{array}\right.
$$

Conversely, suppose that somebody were to give us the words $W_{1}, \ldots, W_{5}$ listed above; then we could write mechanically the sequence $W_{1} b_{1} W_{2} b_{2} W_{3} b_{3} W_{4} b_{4} W_{5}$, after which we could read the result as a legitimate expression defining a functional from $\mathcal{B}^{4}$ to $\mathcal{B}$.

Now, $L$ from the preceding paragraph turns out to be precisely the functional $F^{[\pi]}$ which corresponds to our fixed series $F$ and the noncrossing partition $\pi=$ $\{\{1,4,5\},\{2,3\}\} \in N C(5)$. This is because the words $W_{1}, \ldots, W_{5}$ are exactly 
those created by starting with this special $\pi$ and by applying the rules described in the next definition.

Definition 3.3. Let $F$ be a series in $\operatorname{Ser}(\mathcal{B})$ and let $\pi$ be a partition in $N C(n)$. For every $1 \leq m \leq n$ we define a string of symbols, $W_{m}$, according to the following rules.

- If $m$ is the minimum element of block $V$ of $\pi$ with $|V|=k \geq 2$, then $W_{m}:=$ " $F^{[k]}("$.

- If $m$ is the maximum element of block $V$ of $\pi$ with $|V| \geq 2$, then $W_{m}=$ ")" (just a right bracket).

- If $m$ belongs to a block $V$ of $\pi$ where $\min (V)<m<\max (V)$, then $W_{m}=$ "," (just a comma).

- If $m$ forms by itself a singleton block of $\pi$, then $W_{m}=$ " $F^{[1]}$ " (no parentheses or comma besides the occurrence of $F^{[1]}$ ).

The $\mathbb{C}$-multilinear functional $F^{[\pi]}: \mathcal{B}^{n-1} \rightarrow \mathcal{B}$ is then defined as follows: given $b_{1}, \ldots, b_{n-1} \in \mathcal{B}$ we form the string of symbols obtained by concatenating

$$
W_{1} b_{1} W_{2} b_{2} \cdots W_{n-1} b_{n-1} W_{n}
$$

then we read this as a parenthesized expression which produces an element $b \in \mathcal{B}$, and we define $F^{[\pi]}\left(b_{1}, \ldots, b_{n-1}\right)$ to be equal to this $b$.

Remark 3.4. The special case $\pi=1_{n}$ of the above definition leads to the formula

$$
F^{\left[1_{n}\right]}=F^{[n]}, \quad \forall n \geq 1 .
$$

Indeed, in this case, the string of symbols $W_{1} b_{1} W_{2} b_{2} \cdots W_{n-1} b_{n-1} W_{n}$ has $W_{1}=$ " $F^{[n]}\left(\right.$ ", has $W_{n}=$ ")", and all of $W_{2}, \ldots, W_{n-1}$ are commas.

On the other hand for $\pi=0_{n}$ we get the formula

$$
F^{\left[0_{n}\right]}\left(b_{1}, \ldots, b_{n-1}\right)=F^{[1]} b_{1} F^{[1]} b_{2} \cdots F^{[1]} b_{n-1} \cdots F^{[1]}
$$

(holding for every $n \geq 2$ and $b_{1}, \ldots, b_{n-1} \in \mathcal{B}$ ).

Let us show one more concrete example, illustrating how the nestings and concatenations of blocks of $\pi$ generate a parenthesized expression. Suppose that $n=6$ and that $\pi=\{\{1,3,4\},\{2\},\{5,6\}\} \in N C(6)$. Then the list of words $W_{1}, \ldots, W_{6}$ used to define $F^{[\pi]}$ goes like this:

$$
W_{1}=" F^{[3]}\left(", W_{2}=" F^{[1]} ", W_{5}=" F^{[2]}(",\right.
$$

while $W_{4}$ and $W_{6}$ are right parentheses and $W_{3}$ is a comma. So the string of symbols $W_{1} b_{1} \cdots W_{5} b_{5} W_{6}$ gives us the formula

$$
F^{[\pi]}\left(b_{1}, \ldots, b_{5}\right)=F^{[3]}\left(b_{1} F^{[1]} b_{2}, b_{3}\right) b_{4} F^{[2]}\left(b_{5}\right), \quad \forall b_{1}, \ldots, b_{5} \in \mathcal{B} .
$$

We next record a general fact which follows from the procedure of constructing the functionals $F^{[\pi]}$, and which will be used repeatedly in the sequel.

Lemma 3.5. Let $F$ be a series in $\operatorname{Ser}(\mathcal{B})$ and let $\rho=\left\{V_{1}, \ldots, V_{k}\right\}$ be a partition in $N C(n)$. Consider the formula defining the multilinear functional $F^{[\rho]}$ : $\mathcal{B}^{n-1} \rightarrow \mathcal{B}$. This formula has embedded in it some occurrences of the functionals $F^{\left[\left|V_{1}\right|\right]}, \ldots, F^{\left[\left|V_{k}\right|\right]}$. Suppose that we take some partitions $\pi_{1} \in N C\left(\left|V_{1}\right|\right), \ldots, \pi_{k} \in$ $N C\left(\left|V_{k}\right|\right)$, and that for every $1 \leq j \leq k$ we replace the functional $F^{\left[\left|V_{j}\right|\right]}$ by the functional $F^{\left[\pi_{j}\right]}$, inside the formula for $F^{[\rho]}$. Then the formula defining $F^{[\rho]}$ is transformed into the formula for $F^{[\pi]}$, with $\pi \in N C(n)$ defined as follows:

$$
\pi=\widehat{\pi_{1}} \cup \cdots \cup \widehat{\pi_{k}}
$$


where, for every $1 \leq j \leq k$, we denote by $\widehat{\pi}_{j} \in N C\left(V_{j}\right)$ the noncrossing partition obtained by relabelling $\pi_{j}$.

Proof. Look at the string of symbols $W_{1} b_{1} \cdots W_{n-1} b_{n-1} W_{n}$, which is used in the definition of $F_{\rho}$, and follow how the words $W_{1}, \ldots, W_{n}$ are changed when one replaces every $F^{\left[\left|V_{j}\right|\right]}$ by $F^{\left[\pi_{j}\right]}, 1 \leq j \leq k$. It is immediate that the ensuing string of words is exactly the one which appears in the definition of $F^{[\pi]}$.

Remark 3.6. At some points throughout the paper we will need a variation of the construction of $F^{[\pi]}$ which involves coloured noncrossing partitions. For the sake of simplicity, here we discuss the situation of colourings which use two colours. Given a partition $\pi \in N C(n)$, a colouring of $\pi$ is then a map $c: \pi \rightarrow\{1,2\}$ (that is, a procedure which associates to every block $V$ of $\pi$ a number $c(V) \in\{1,2\})$. For such $\pi$ and $c$ one can talk about "mixed nested functionals" of the form

$$
(F, G)^{[\pi, c]} \text {, }
$$

where $F, G$ are two series in $\operatorname{Ser}(\mathcal{B})$. The object in (3.1) is a multilinear functional from $\mathcal{B}^{n-1}$ to $\mathcal{B}$, constructed by the same method as in Definition 3.3. but which uses some terms of $F$ and some terms of $G$ (depending on what is the colour of the corresponding block of $\pi$; blocks of colour 1 go with $F$, and blocks of colour 2 go with $G)$.

Concrete example: Take again the case when $\pi=\{\{1,3,4\},\{2\},\{5,6\}\} \in$ $N C(6)$, as we illustrated at the end of Remark 3.4 Suppose that $\pi$ is coloured so that $c(\{1,3,4\})=1$, while $c(\{2\})=c(\{5,6\})=2$. Then the list of words $W_{1}, \ldots, W_{6}$ that we use is changed in the respect that we now have

$$
W_{1}=\text { " } F^{[3]}\left(", W_{2}=\text { "G } G^{[1] "}, W_{5}=\text { "G } G^{[2]}(",\right.
$$

(while $W_{4}$ and $W_{6}$ still are right parentheses, and $W_{3}$ is a comma). The string of symbols $W_{1} b_{1} \cdots W_{5} b_{5} W_{6}$ thus gives the formula

$$
(F, G)^{[\pi, c]}\left(b_{1}, \ldots, b_{5}\right)=F^{[3]}\left(b_{1} G^{[1]} b_{2}, b_{3}\right) b_{4} G^{[2]}\left(b_{5}\right), \quad \forall b_{1}, \ldots, b_{5} \in \mathcal{B} .
$$

Clearly, the same idea of colouring can be used when more than two colours are involved, in order to define for instance mixed linear functionals of the form

$$
(F, G, H)^{[\pi, c]},
$$

where $F, G, H$ are series in $\operatorname{Ser}(\mathcal{B}), \pi$ is in $N C(n)$, and $c: \pi \rightarrow\{1,2,3\}$ is a colouring of $\pi$ in three colours.

Remark 3.7 (Interval partitions). At various points in the paper we will need to look at functionals $F^{[\pi]}$ (as introduced in Definition [3.3) in the special, simpler, case when $\pi$ is an interval partition. Here we record the formula that is relevant for such a special case.

A partition $\pi=\left\{V_{1}, \ldots, V_{k}\right\}$ of $\{1, \ldots, n\}$ is said to be an interval partition when every block $V_{i}$ is of the form $[p, q] \cap \mathbb{Z}$ for some $1 \leq p \leq q \leq n$. The set of all interval partitions of $\{1, \ldots, n\}$ will be denoted as $\operatorname{Int}(n)$. It is clear that $\operatorname{Int}(n) \subseteq N C(n)$, but it is occasionally preferable to think of $\operatorname{Int}(n)$ as a partially ordered set in its own right, with partial order " $\leq$ " still given by reverse refinement. It is easily seen that $(\operatorname{Int}(n), \leq)$ is then isomorphic to the partially ordered set of subsets of $\{1, \ldots, n-1\}$.

Now let $F$ be in $\operatorname{Ser}(\mathcal{B})$ and let $\pi$ be a partition in $\operatorname{Int}(n)$. Let us write explicitly $\pi=\left\{V_{1}, \ldots, V_{k}\right\}$, with the blocks $V_{i}$ picked so that $\min \left(V_{1}\right)<\min \left(V_{2}\right)<\cdots<$ 
$\min \left(V_{k}\right)$. Consider the numbers $1 \leq q_{1}<q_{2}<\cdots<q_{k}=n$ obtained by putting

$$
q_{i}=\left|V_{1}\right|+\left|V_{2}\right|+\cdots+\left|V_{i}\right|, \quad 1 \leq i \leq k .
$$

It is then immediate that the multilinear functional $F^{[\pi]}: \mathcal{B}^{n-1} \rightarrow \mathcal{B}$ acts by

$$
\begin{gathered}
F^{[\pi]}\left(b_{1}, \ldots, b_{n-1}\right)=F^{\left[q_{1}\right]}\left(b_{1}, \ldots, b_{q_{1}-1}\right) b_{q_{1}} \\
\times F^{\left[q_{2}-q_{1}\right]}\left(b_{q_{1}+1}, \ldots, b_{q_{2}-1}\right) b_{q_{2}} \cdots b_{q_{k-1}} F^{\left[q_{k}-q_{k-1}\right]}\left(b_{q_{k-1}+1}, \ldots, b_{q_{k}-1}\right),
\end{gathered}
$$

for $b_{1}, \ldots, b_{n-1} \in \mathcal{B}$.

\section{4. $R$-Transform, $B$-Transform, AND CONVOLUtion POWERS}

In Section 2 we saw that the map $\mu \mapsto M_{\mu}$ is a bijection from $\Sigma_{\text {alg }}(\mathcal{B})$ onto $\operatorname{Ser}(\mathcal{B})$. In this section we will review two other important bijections from $\Sigma_{\text {alg }}(\mathcal{B})$ onto $\operatorname{Ser}(\mathcal{B})$, which associate to every $\mu$ its $R$-transform and its $B$-transform. Both these bijections can be treated combinatorially by using some bijective self-maps of $\operatorname{Ser}(\mathcal{B})$ : one bijection which connects the moment series $M_{\mu}$ to the $R$-transform $R_{\mu}$, and another bijection which connects the moment series $M_{\mu}$ to the $B$-transform $B_{\mu}, \mu \in \Sigma_{\text {alg }}(\mathcal{B})$. For lack of better names, we will use the notation $\widehat{\mathrm{RM}}$ and respectively $\widehat{\mathrm{BM}}$ for these two self-maps of $\operatorname{Ser}(\mathcal{B})$. It is useful that $\widehat{\mathrm{RM}}$ and $\widehat{\mathrm{BM}}$ can be introduced by explicit summation formulas which don't make reference to distributions, as follows.

Notation 4.1. For $F \in \operatorname{Ser}(\mathcal{B})$ we denote by $\widehat{\mathrm{RM}}(F)$ the series $G \in \operatorname{Ser}(\mathcal{B})$ with terms defined as follows:

$$
G^{[n]}:=\sum_{\pi \in N C(n)} F^{[\pi]}, \quad \forall n \geq 1 .
$$

Remark 4.2. By making appropriate use of Lemma 3.5, one finds that (4.1) extends to the formula

$$
G^{[\rho]}=\sum_{\substack{\pi \in N C(n), \pi \leq \rho}} F^{[\pi]},
$$

holding for all $n \geq 1$ and $\rho \in N C(n)$. One then invokes the Möbius inversion formula for the poset $N C(n)$ in order to invert (4.2); this leads to the formula

$$
F^{[\rho]}=\sum_{\substack{\pi \in N C(n), \pi \leq \rho}} \operatorname{Moeb}(\pi, \rho) G^{[\pi]},
$$

holding for $n \geq 1$ and $\rho \in N C(n)$, and where "Moeb" stands for the Möbius function of $N C(n)$ (see e.g. the review of Moeb made in Lecture 10 of [12]). In particular, the terms of the series $F$ can be recaptured via the formula

$$
F^{[n]}=\sum_{\pi \in N C(n)} \operatorname{Moeb}\left(\pi, 1_{n}\right) G^{[\pi]}, \quad \forall n \geq 1 .
$$

From here one immediately finds that the map $\widehat{\mathrm{RM}}: \operatorname{Ser}(\mathcal{B}) \rightarrow \operatorname{Ser}(\mathcal{B})$ is a bijection, having for inverse the map $G \mapsto F$ described by (4.4). 
Definition 4.3. For every distribution $\mu \in \Sigma_{\text {alg }}(\mathcal{B})$, the series

$$
R_{\mu}:=\widehat{\mathrm{RM}}^{-1}\left(M_{\mu}\right) \in \operatorname{Ser}(\mathcal{B})
$$

is called the $R$-transform of $\mu$.

The construction of the bijection $\widehat{\mathrm{BM}}$ and the definition of the $B$-transform associated to a distribution $\mu \in \Sigma_{\text {alg }}(\mathcal{B})$ are done in exactly the same way, but where now instead of $N C(n)$ one uses the smaller poset $\operatorname{Int}(n)$ of interval partitions.

Notation 4.4. For $F \in \operatorname{Ser}(\mathcal{B})$ we denote by $\widehat{\mathrm{BM}}(F)$ the series $G \in \operatorname{Ser}(\mathcal{B})$ with terms defined as follows:

$$
G^{[n]}:=\sum_{\pi \in \operatorname{Int}(n)} F^{[\pi]}, \quad \forall n \geq 1 .
$$

Remark 4.5. Exactly as in Remark 4.2, one sees that (4.6) extends to the formula

$$
G^{[\rho]}=\sum_{\substack{\pi \in \operatorname{Int}(n), \pi \leq \rho}} F^{[\pi]},
$$

holding for all $n \geq 1$ and $\rho \in \operatorname{Int}(n)$. One then uses Möbius inversion in the poset $\operatorname{Int}(n)$ in order to invert (4.7). Since $\operatorname{Int}(n)$ is isomorphic to a Boolean poset, this inversion process is in fact quite straightforward and leads to the formula

$$
F^{[\rho]}=\sum_{\substack{\pi \in \operatorname{Int}(n), \pi \leq \rho}}(-1)^{|\pi|-|\rho|} G^{[\pi]},
$$

holding for $n \geq 1$ and $\rho \in \operatorname{Int}(n)$. In particular, the terms of the series $F$ are recaptured from $G$ via the formula

$$
F^{[n]}=\sum_{\pi \in \operatorname{Int}(n)}(-1)^{|\pi|-1} G^{[\pi]}, \quad \forall n \geq 1 .
$$

In this way it becomes clear that the map $\widehat{\mathrm{BM}}: \operatorname{Ser}(\mathcal{B}) \rightarrow \operatorname{Ser}(\mathcal{B})$ is a bijection, having for inverse the map $G \mapsto F$ described by (4.9).

Definition 4.6. For every distribution $\mu \in \Sigma_{a l g}(\mathcal{B})$, the series

$$
B_{\mu}:=\widehat{\mathrm{BM}}^{-1}\left(M_{\mu}\right) \in \operatorname{Ser}(\mathcal{B})
$$

is called the $B$-transform of $\mu$. Note that in many sources this would be called "the $\eta$-series of $\mu "$, but we reserve the letter $\eta$ for maps and covariances.

Now, with definitions laid out as above, there is no problem to define generalized convolution powers with respect to $\boxplus$ and $\uplus$, as follows.

Definition 4.7. Let $\mu$ be a distribution in $\Sigma_{\text {alg }}(\mathcal{B})$, and let $\alpha: \mathcal{B} \rightarrow \mathcal{B}$ be a linear map.

$1^{o}$ We will denote by $\mu^{\boxplus \alpha}$ the distribution in $\Sigma_{a l g}(\mathcal{B})$ which is uniquely determined by the fact that its $R$-transform is

$$
R_{\mu^{\boxplus \alpha}}=\alpha \circ R_{\mu} .
$$


$2^{o}$ We will denote by $\mu^{\uplus \alpha}$ the distribution in $\Sigma_{a l g}(\mathcal{B})$ which is uniquely determined by the fact that its $B$-series is

$$
B_{\mu}^{\uplus \alpha}=\alpha \circ B_{\mu} .
$$

Remark 4.8. Directly from the above definition, we have semigroup properties for each of the two types of convolution powers. More precisely: for every $\mu \in \Sigma_{\text {alg }}(\mathcal{B})$ and all linear maps $\alpha, \beta: \mathcal{B} \rightarrow \mathcal{B}$ we have

$$
\left(\mu^{\boxplus \alpha}\right)^{\boxplus \beta}=\mu^{\boxplus(\beta \circ \alpha)} \text { and }\left(\mu^{\uplus \alpha}\right)^{\uplus \beta}=\mu^{\uplus(\beta \circ \alpha)} .
$$

\section{The BiJeCtion CONNECTING $R$-TRAnsform to $B$-TRANSFORM}

We continue to use the framework from the preceding two sections. We will now examine another bijective self-map of $\operatorname{Ser}(\mathcal{B})$, which combines the two bijections $R_{\mu} \mapsto M_{\mu}$ and $B_{\mu} \mapsto M_{\mu}$ discussed in Section 4 , and acts by the prescription that

$$
R_{\mu} \mapsto B_{\mu}, \quad \mu \in \Sigma_{a l g}(\mathcal{B}) .
$$

It is useful that this bijection can be introduced by a direct combinatorial formula, without making explicit reference to the transforms $R$ and $B$. The direct formula will be given in Definition 5.2 then the " $R_{\mu} \mapsto B_{\mu}$ " property will be derived in Proposition 5.4.

In order to state Definition [5.2, we first review a few more details of the combinatorics of $N C(n)$.

Remark 5.1 (The partial order $\ll$ in $N C(n)$ ). For $\pi, \rho \in N C(n)$ we will write " $\pi \ll \rho "$ to mean that $\pi \leq \rho$ and that, in addition, the following condition is fulfilled:

$$
\left\{\begin{array}{l}
\text { For every block } W \text { of } \rho \text { there exists a block } \\
V \text { of } \pi \text { such that } \min (W), \max (W) \in V
\end{array}\right.
$$

It is immediately verified that "«" is a partial order relation on $N C(n)$. It is much coarser than the reverse refinement order and differs from it in several respects. In particular, observe that $(N C(n), \ll)$ has many maximal elements: they are precisely the interval partitions, and for every $\pi \in N C(n)$ there exists a unique interval partition $\rho$ such that $\pi \ll \rho$. (The blocks of this unique interval partition $\rho$ are in some sense the convex hulls of the outer blocks of $\pi$.)

A special role in the subsequent calculation will be played by the partitions $\pi \in N C(n)$ such that $\pi \ll 1_{n}$. The latter inequality means (obvious from the definition) that $\pi$ has a unique outer block, which will be denoted by $V_{o}(\pi)$.

Definition 5.2. We define a map $\widehat{\mathrm{RB}}: \operatorname{Ser}(\mathcal{B}) \rightarrow \operatorname{Ser}(\mathcal{B})$ in the following way: for every $F \in \operatorname{Ser}(\mathcal{B})$ we put $\widehat{\mathrm{RB}}(F)$ to be the series $G \in \operatorname{Ser}(\mathcal{B})$ with

$$
G^{[n]}=\sum_{\substack{\pi \in N C(n) \\ \pi \ll 1_{n}}} F^{[\pi]}, \quad \forall n \geq 1 .
$$


Remark 5.3. $1^{\circ}$ It is useful to once again invoke Lemma 3.5 in order to note that (5.2) extends to the formula

$$
G^{[\rho]}=\sum_{\substack{\pi \in N C(n) \\ \pi \ll \rho}} F^{[\pi]},
$$

holding for every $n \geq 1$ and every $\rho \in N C(n)$.

$2^{o}$ The notation " $\widehat{\mathrm{RB}}$ " used in Definition 5.2 is meant to be suggestive of the fact that we are dealing with the map which "converts the $R$-transform into the $B$ transform" (as will be proved in the next proposition). This map and its properties were previously studied in 2, 4, in the framework of multi-variable distributions over $\mathbb{C}$. A comment on notation: the papers [2, 4 use the fairly widespread name of " $\eta$-series" for the $B$-transform of a distribution $\mu$; as a consequence, the map which connects the transforms is called in those papers by the more sonorous name of "Reta", rather than $\widehat{\mathrm{RB}}$.

Proposition 5.4. The maps $\widehat{R M}, \widehat{B M}$ from Section 4 and the map $\widehat{R B}$ from Definition 5.2 are related by the formula

$$
\widehat{B M} \circ \widehat{R B}=\widehat{R M} .
$$

As a consequence, it follows that $\widehat{R B}$ is a bijection from $\operatorname{Ser}(\mathcal{B})$ onto itself and has the property that

$$
\widehat{R B}\left(R_{\mu}\right)=B_{\mu}, \quad \forall \mu \in \Sigma_{\text {alg }}(\mathcal{B}) .
$$

Proof. For the verification of (5.4) let us consider a series $F \in \operatorname{Ser}(\mathcal{B})$ and let us make the notation

$$
\widehat{\mathrm{RB}}(F)=: G \text {, then } \widehat{\mathrm{BM}}(G)=: H .
$$

We have to prove that $H$ is equal to $\widehat{\mathrm{RM}}(F)$. In order to verify this, we pick a positive integer $n$ and we calculate:

$$
\begin{aligned}
H^{[n]} & \left.=\sum_{\rho \in \operatorname{Int}(n)} G^{[\rho]} \quad \text { (by the definition of } \widehat{\mathrm{BM}}\right) \\
& =\sum_{\rho \in \operatorname{Int}(n)}\left(\sum_{\substack{\pi \in N C(n) \\
\pi \ll \rho}} F^{[\pi]}\right) \quad(\text { by }(\underline{5.3}) .
\end{aligned}
$$

We next observe that the double sum which has appeared is in fact just a sum over $\pi \in N C(n)$; this is due to the observation, recorded in Remark 5.1, that for every $\pi \in N C(n)$ there exists a unique $\rho \in \operatorname{Int}(n)$ such that $\pi \ll \rho$. So then our calculation for $H^{[n]}$ becomes

$$
H^{[n]}=\sum_{\pi \in N C(n)} F^{[\pi]}=(\widehat{\operatorname{RM}}(F))^{[n]},
$$

and the equality $\widehat{\mathrm{RM}}(F)=H$ follows.

Since we saw in Section 4 that each of the maps $\widehat{\mathrm{RM}}$ and $\widehat{\mathrm{BM}}$ is a bijection from $\operatorname{Ser}(\mathcal{B})$ to itself, the formula obtained in (5.4) implies that $\widehat{\mathrm{RB}}=\widehat{\mathrm{BM}}^{-1} \circ \widehat{\mathrm{RM}}$ has this property as well. 
Finally, in order to obtain (5.5) we write

$$
\begin{aligned}
\widehat{\mathrm{BM}}\left(\widehat{\mathrm{RB}}\left(R_{\mu}\right)\right) & \left.=\widehat{\mathrm{RM}}\left(R_{\mu}\right) \quad \text { (by }(\underline{5.4})\right) \\
& \left.=M_{\mu} \quad \text { (by the definition of } \widehat{\mathrm{RM}}\right) \\
& \left.=\widehat{\mathrm{BM}}\left(B_{\mu}\right) \quad \text { (by the definition of } \widehat{\mathrm{BM}}\right)
\end{aligned}
$$

Since $\widehat{\mathrm{BM}}$ is one-to-one, it follows that $\widehat{\mathrm{RB}}\left(R_{\mu}\right)=B_{\mu}$, as claimed.

In the discussion about the transformations $\mathbb{B}_{\alpha}$ in the next section, we will need to extend Definition 5.2 to a family of bijective maps $\widehat{\mathrm{RB}}_{\alpha}: \operatorname{Ser}(\mathcal{B}) \rightarrow \operatorname{Ser}(\mathcal{B})$, where $\alpha$ runs in the set of linear transformations from $\mathcal{B}$ to $\mathcal{B}$. The original $\widehat{\mathrm{RB}}$ from Definition 5.2 will correspond to the special case when $\alpha$ is the identity transformation of $\mathcal{B}$. The definition of $\widehat{\mathrm{RB}}_{\alpha}(F)$ is obtained by substituting $\alpha \circ F$ instead of $F$ on the right-hand side of (5.2), but where we make one important exception to this substitution rule: for every $\pi \in N C(n)$ such that $\pi \ll 1_{n}$, the unique outer block of $\pi$ still carries with it a term of the series $F$ (not substituted by the corresponding term of $\alpha \circ F)$. Because of this exception, the formal definition of $\widehat{\mathrm{RB}}_{\alpha}(F)$ will thus be phrased in terms of colourings of noncrossing partitions, as discussed in Remark 3.6. Specifically, we will use the colouring of $\pi \ll 1_{n}$, where the unique outer block $V_{o}(\pi)$ of $\pi$ is coloured differently from the other blocks.

Definition 5.5. $1^{o}$ Let $\pi$ be a partition in $N C(n)$ such that $\pi \ll 1_{n}$. We will denote by $\mathrm{o}_{\pi}$ the colouring of $\pi$ defined by

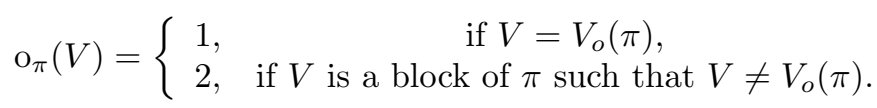

$2^{o}$ Let $\alpha: \mathcal{B} \rightarrow \mathcal{B}$ be a linear transformation. We define a map $\widehat{\mathrm{RB}}_{\alpha}: \operatorname{Ser}(\mathcal{B}) \rightarrow$ $\operatorname{Ser}(\mathcal{B})$ in the following way: for every $F \in \operatorname{Ser}(\mathcal{B})$ we put $\widehat{\mathrm{RB}}_{\alpha}(F)$ to be the series $G \in \operatorname{Ser}(\mathcal{B})$ with

$$
G^{[n]}=\sum_{\substack{\pi \in N C(n) \\ \pi \ll 1_{n}}}(F, \alpha \circ F)^{\left[\pi, \mathrm{O}_{\pi}\right]}, \quad \forall n \geq 1
$$

(and where the right-hand side of (5.6) follows the notation introduced in Remark (3.6).

Remark 5.6. $1^{o}$ In order to get a better idea about how $\widehat{\mathrm{RB}}_{\alpha}$ works, let us write explicitly what (5.6) becomes for some small values of $n$. We have

$$
\begin{gathered}
G^{[1]}=F^{[1]}, \quad G^{[2]}(b)=F^{[2]}(b) \\
G^{[3]}\left(b_{1}, b_{2}\right)=F^{[3]}\left(b_{1}, b_{2}\right)+F^{[2]}\left(b_{1} \alpha\left(F^{[1]}\right) b_{2}\right) \\
G^{[4]}\left(b_{1}, b_{2}, b_{3}\right)=F^{[4]}\left(b_{1}, b_{2}, b_{3}\right)+F^{[3]}\left(b_{1} \alpha\left(F^{[1]}\right) b_{2}, b_{3}\right) \\
+F^{[3]}\left(b_{1}, b_{2} \alpha\left(F^{[1]}\right) b_{3}\right)+F^{[2]}\left(b_{1}\left(\alpha \circ F^{[2]}\right)\left(b_{2}\right) b_{3}\right) \\
+F^{[2]}\left(b_{1} \alpha\left(F^{[1]}\right) b_{2} \alpha\left(F^{[1]}\right) b_{3}\right) .
\end{gathered}
$$

$2^{o}$ From the above definitions it is immediate that the map $\widehat{\mathrm{RB}}$ from Definition 5.2 becomes $\widehat{\mathrm{RB}}_{1}$, where $1: \mathcal{B} \rightarrow \mathcal{B}$ is the identity. Let us also note that if we denote 
by $0: \mathcal{B} \rightarrow \mathcal{B}$ the map which is identically equal to 0 , then $\widehat{\mathrm{RB}}_{0}$ is the identity map on $\operatorname{Ser}(\mathcal{B})$ (indeed, in the sum on the right-hand side of $(5.6)$ the only term which survives is the one indexed by $1_{n}$, and thus we get $G^{[n]}=F^{[n]}$ for every $n \geq 1$ ).

$3^{o}$ Clearly, the definition of $\widehat{\mathrm{RB}}_{\alpha}$ was made in such a way that we have

$$
\alpha \circ \widehat{\mathrm{RB}}_{\alpha}(F)=\widehat{\mathrm{RB}}(\alpha \circ F), \quad \forall F \in \operatorname{Ser}(\mathcal{B}) .
$$

In the case when $\alpha$ is invertible, one can thus introduce $\widehat{\mathrm{RB}}_{\alpha}$ by the simpler formula

$$
\widehat{\mathrm{RB}}_{\alpha}(F)=\alpha^{-1} \circ \widehat{\mathrm{RB}}(\alpha \circ F), \quad F \in \operatorname{Ser}(\mathcal{B}) .
$$

The summation formula used in (5.6) is more tortuous, but has the merit that it works without assuming that $\alpha$ is invertible.

$4^{o}$ The main point we want to make about the maps $\widehat{\mathrm{RB}}_{\alpha}$ is that they form a commutative semigroup under composition. This is stated precisely in Proposition 5.9 below. In the proof of Proposition 5.9 we will use an important property of the partial order $\ll$, reviewed in Proposition 5.8, which essentially says that $\ll$ has "some Boolean lattice features" embedded into it.

Definition 5.7. Let $\pi, \rho$ be partitions in $N C(n)$ such that $\pi \ll \rho$. A block $V$ of $\pi$ is said to be $\rho$-special when there exists a block $W$ of $\rho$ such that $\min (V)=\min (W)$ and $\max (V)=\max (W)$.

Proposition 5.8. Let $\pi \in N C(n)$ be such that $\pi \ll 1_{n}$ and consider the set of partitions

$$
\left\{\rho \in N C(n) \mid \pi \ll \rho \ll 1_{n}\right\} .
$$

Then $\rho \mapsto\{V \in \pi \mid V$ is $\rho$-special $\}$ is a one-to-one map from the set (5.9) to the set of subsets of $\pi$. The image of this map is equal to $\left\{\mathfrak{V} \subseteq \pi \mid \mathfrak{V} \ni V_{o}(\pi)\right\}$.

For the proof of Proposition 5.8, the reader is referred to Proposition 2.13 and Remark 2.14 of [2].

Proposition 5.9. For any linear transformations $\alpha, \beta: \mathcal{B} \rightarrow \mathcal{B}$, one has that

$$
\widehat{R B}_{\alpha} \circ \widehat{R B}_{\beta}=\widehat{R B}_{\alpha+\beta} \text {. }
$$

Proof. Let $F$ be a series in $\operatorname{Ser}(\mathcal{B})$, and let us denote $\widehat{\mathrm{RB}}_{\beta}(F)=: G, \widehat{\mathrm{RB}}_{\alpha}(G)=: H$. We have to prove that $H=\widehat{\mathrm{RB}}_{\alpha+\beta}(F)$. For the whole proof we fix a positive integer $n$, for which we will verify that the $n$-th term of $H$ is equal to the $n$-th term of $\widehat{\mathrm{RB}}_{\alpha+\beta}(F)$.

From the definition of $\widehat{\mathrm{RB}}_{\beta}$ it follows that we have

$$
H^{[n]}=\sum_{\substack{\rho \in N C(n), \rho \ll 1_{n}}}(G, \beta \circ G)^{\left[\rho, \mathrm{O}_{\rho}\right]} .
$$

Fix for the moment a partition $\rho \in N C(n)$ such that $\rho \ll 1_{n}$. Let us write explicitly $\rho=\left\{V_{1}, \ldots, V_{k}\right\}$, where $V_{1}$ is the block which contains $1, n$. Then $(G, \beta \circ$ $G)^{\left[\rho, \mathrm{O}_{\rho}\right]}$ is a multilinear functional from $\mathcal{B}^{n-1}$ to $\mathcal{B}$, and its explicit description involves the functionals

$$
G^{\left[\left|V_{1}\right|\right]} \text { and } \beta \circ G^{\left[\left|V_{2}\right|\right]}, \ldots, \beta \circ G^{\left[\left|V_{k}\right|\right]}
$$


nested in various ways (with each of these functionals used exactly once, and with $G_{\left|V_{1}\right|}$ appearing "on the outside"). Let us next replace each of $G^{\left[\left|V_{1}\right|\right]}, \ldots, G^{\left[\left|V_{k}\right|\right]}$ from how they are defined (in reference to the terms of $F$ and of $\alpha \circ F$ ) in (5.6). This gives us the functional $(G, \beta \circ G)^{[\rho]}$ expressed as a sum of the form

$$
(G, \beta \circ G)^{\left[\rho, \mathrm{O}_{\rho}\right]}=\sum_{\substack{\pi \in N C(n), \pi \ll \rho}} \operatorname{term}_{\pi},
$$

where every functional term $\pi: \mathcal{B}^{n-1} \rightarrow \mathcal{B}$ is obtained by nesting (in the way dictated by the nestings of blocks of $\pi$ ) some terms of the series $F, \alpha \circ F$ and $\beta \circ F$. (It is important to note here that, because of how our definitions are run, we never get to deal with terms of the functional $\alpha \circ \beta \circ F$.) A moment's thought shows in fact that the precise formula for term ${ }_{\pi}$ is

$$
\operatorname{term}_{\pi}=(F, \alpha \circ F, \beta \circ F)^{\left[\pi, c_{\pi, \rho}\right]},
$$

where the colouring $c_{\pi, \rho}$ of $\pi$ goes in the way described as follows: we colour a general block $V$ of $\pi$ by putting

$$
c_{\pi, \rho}(V)= \begin{cases}1, & \text { if } V=V_{o}(\pi) \\ 2, & \text { if } V \text { is } \rho \text {-special but } V \neq V_{o}(\pi) \\ 3, & \text { if } V \text { is not } \rho \text {-special. }\end{cases}
$$

Returning to the formula for $H^{[n]}$, we have thus obtained that

$$
H^{[n]}=\sum_{\substack{\rho \in N C(n), \rho \ll 1_{n}}}\left(\sum_{\substack{\pi \in N C(n), \pi \ll \rho}}(F, \alpha \circ F, \beta \circ F)^{\left[\pi, c_{\pi, \rho}\right]}\right) .
$$

Changing the order of summation, this becomes

$$
H^{[n]}=\sum_{\substack{\pi \in N C(n), \pi \ll 1_{n}}}\left(\sum_{\substack{\rho \in N C(n), \\ \text { such that } \\ \pi \ll \rho \ll 1_{n}}}(F, \alpha \circ F, \beta \circ F)^{\left[\pi, c_{\pi, \rho}\right]}\right) .
$$

Fix $\pi$ and use the parametrization of $\left\{\rho \in N C(n) \mid \pi \ll \rho \ll 1_{n}\right\}$ provided by Proposition [5.8. Then group together $\alpha$ 's and $\beta$ 's into occurrences of $\alpha+\beta$. We arrive exactly at the description for the $n$-th term of the series $\widehat{\mathrm{RB}}_{\alpha+\beta}(F)$.

Corollary 5.10. Let $\alpha: \mathcal{B} \rightarrow \mathcal{B}$ be a linear transformation. The map $\widehat{R B}_{\alpha}$ : $\operatorname{Ser}(\mathcal{B}) \rightarrow \operatorname{Ser}(\mathcal{B})$ is bijective and has inverse equal to $\widehat{R B}_{-\alpha}$.

Proof. This is immediate from Proposition 5.9 and the fact that $\widehat{\mathrm{RB}}_{0}$ is the identity map on $\operatorname{Ser}(\mathcal{B})$. 
Remark 5.11. From the above corollary we get in particular an explicit formula for the inverse of the original bijection $\widehat{\mathrm{RB}}$ from Definition 5.2 . Indeed, this inverse is

$$
\widehat{\mathrm{RB}}^{-1}=\widehat{\mathrm{RB}}_{1}^{-1}=\widehat{\mathrm{RB}}_{-1},
$$

with $(-1): \mathcal{B} \rightarrow \mathcal{B}$ being the map $b \mapsto-b$. But when we invoke (5.8) in the special case of $\alpha=-1$, the series $\alpha^{-1} \circ \widehat{\mathrm{RB}}(\alpha \circ F)$ from its right-hand side simply becomes $-\widehat{\mathrm{RB}}(-F)$. We are thus led to the conclusion that the inverse of $\widehat{\mathrm{RB}}$ acts by

$$
\widehat{\mathrm{RB}}^{-1}(F)=-\widehat{\mathrm{RB}}(-F), \quad \forall F \in \operatorname{Ser}(\mathcal{B}) .
$$

\section{The transformations $\mathbb{B}_{\boldsymbol{\alpha}}$}

In this section we continue to use the framework and notation considered in Sections $3-5$.

Definition 6.1. The Boolean-to-free Bercovici-Pata bijection is the map $\mathbb{B}: \Sigma_{\text {alg }}(\mathcal{B})$ $\rightarrow \Sigma_{\text {alg }}(\mathcal{B})$ defined via the requirement that

$$
R_{\mathbb{B}[\mu]}=B_{\mu}, \quad \text { for } \mu \in \Sigma_{a l g}(\mathcal{B}) .
$$

Remark 6.2. From the discussion in Section 4 it is clear that $\mathbb{B}$ is indeed a bijection from $\Sigma_{\text {alg }}(\mathcal{B})$ to itself; indeed, one can write $\mathbb{B}=\underline{R}^{-1} \circ \underline{B}$, where the bijections $\underline{R}, \underline{B}: \Sigma_{\text {alg }}(\mathcal{B}) \rightarrow \operatorname{Ser}(\mathcal{B})$ are defined by sending $\mu \mapsto R_{\mu}$ and respectively $\mu \mapsto B_{\mu}$, for $\left.\mu \in \Sigma_{\text {alg }}(\mathcal{B})\right)$. The bijection $\mathbb{B}$ is important because it has meaning in the analytic framework, where it sends general distributions to $\boxplus$-infinitely divisible distributions. (In the $\mathbb{C}$-valued framework, this was found by Bercovici and Pata [8]. The $\mathcal{B}$-valued version of the result was recently established in [7].)

In this section we show how the bijection $\mathbb{B}$ from Definition 6.1 is incorporated into a semigroup of bijective transformations of $\Sigma_{\text {alg }}(\mathcal{B})$, defined as follows.

Definition 6.3. Let $\mu$ be a distribution in $\Sigma_{\text {alg }}(\mathcal{B})$ and let $\alpha: \mathcal{B} \rightarrow \mathcal{B}$ be a linear map. We define a new distribution $\mathbb{B}_{\alpha}(\mu) \in \Sigma_{\text {alg }}(\mathcal{B})$ by requiring that its $R$-transform is

$$
R_{\mathbb{B}_{\alpha}(\mu)}=\widehat{\mathrm{RB}}_{\alpha}\left(R_{\mu}\right) .
$$

In this way, for every fixed $\alpha$ we get a map $\mathbb{B}_{\alpha}: \Sigma_{\text {alg }}(\mathcal{B}) \rightarrow \Sigma_{\text {alg }}(\mathcal{B})$.

Theorem 6.4. $1^{o}$ For any two linear transformations $\alpha, \beta: \mathcal{B} \rightarrow \mathcal{B}$ one has that

$$
\mathbb{B}_{\alpha} \circ \mathbb{B}_{\beta}=\mathbb{B}_{\alpha+\beta} .
$$

$2^{\circ}$ Let $0: \mathcal{B} \rightarrow \mathcal{B}$ be the linear transformation which is identically equal to 0 . Then $\mathbb{B}_{0}$ is the identity map on $\Sigma_{\text {alg }}(\mathcal{B})$.

$3^{\circ}$ For every linear transformation $\alpha: \mathcal{B} \rightarrow \mathcal{B}$, the map $\mathbb{B}_{\alpha}: \Sigma_{\text {alg }}(\mathcal{B}) \rightarrow \Sigma_{\text {alg }}(\mathcal{B})$ is bijective, with inverse equal to $\mathbb{B}_{-\alpha}$.

$4^{\circ}$ Let $1: \mathcal{B} \rightarrow \mathcal{B}$ be the identity transformation. Then $\mathbb{B}_{1}=\mathbb{B}$ (the BercoviciPata bijection reviewed in Definition 6.1).

Proof. In order to prove $1^{o}$, let us fix a $\mu \in \Sigma_{\text {alg }}(\mathcal{B})$ and show that

$$
\mathbb{B}_{\alpha}\left(\mathbb{B}_{\beta}(\mu)\right)=\mathbb{B}_{\alpha+\beta}(\mu) .
$$


We do this by verifying that the distributions on the two sides of (6.4) have the same $R$-transform. Indeed, by starting from the left-hand side we can write

$$
\begin{aligned}
R_{\mathbb{B}_{\alpha}\left(\mathbb{B}_{\beta}(\mu)\right)} & =\widehat{\mathrm{RB}}_{\alpha}\left(R_{\mathbb{B}_{\beta}(\mu)}\right) \\
& =\widehat{\mathrm{RB}}_{\alpha}\left(\widehat{\mathrm{RB}}_{\beta}\left(R_{\mu}\right)\right) \\
& =\widehat{\mathrm{RB}}_{\alpha+\beta}\left(R_{\mu}\right) \text { (by Proposition [5.9) } \\
& =R_{\mathbb{B}_{\alpha+\beta}(\mu)} .
\end{aligned}
$$

Statement $2^{o}$ is immediate from the fact that $\widehat{\mathrm{RB}}_{0}$ is the identity map on $\operatorname{Ser}(\mathcal{B})$, and $3^{\circ}$ is an immediate consequence of $1^{\circ}$ and $2^{\circ}$.

Finally, for $4^{o}$ let us fix a $\mu \in \Sigma_{\text {alg }}(\mathcal{B})$ for which we prove that $\mathbb{B}_{1}(\mu)=\mathbb{B}(\mu)$. We do this by verifying that the two distributions in question have the same $R$ transform:

$$
\begin{aligned}
& R_{\mathbb{B}_{1}(\mu)}=\widehat{\mathrm{RB}}_{1}\left(R_{\mu}\right) \\
& =\widehat{\mathrm{RB}}\left(R_{\mu}\right) \text { (since } \widehat{\mathrm{RB}}_{1}=\widehat{\mathrm{RB}} \text { ) } \\
& =B_{\mu} \text { (by Proposition 5.4) } \\
& =R_{\mathbb{B}(\mu)} \text {. }
\end{aligned}
$$

In Definition 6.3, the distribution $\mathbb{B}_{\alpha}(\mu)$ was introduced via a description of its $R$-transform. Next we observe that we could have come to the same definition by a very similar description phrased in terms of $B$-transforms.

Proposition 6.5. For every $\mu \in \Sigma_{\text {alg }}(\mathcal{B})$ and every linear transformation $\alpha: \mathcal{B} \rightarrow$ $\mathcal{B}$ one has that

$$
B_{\mathbb{B}_{\alpha}(\mu)}=\widehat{R B}_{\alpha}\left(B_{\mu}\right)
$$

Proof. We calculate:

$$
\begin{aligned}
B_{\mathbb{B}_{\alpha}(\mu)} & =R_{\left.\mathbb{B}_{(} \mathbb{B}_{\alpha}(\mu)\right)} \\
& =R_{\mathbb{B}_{\alpha+1}(\mu)}\left(\text { by } 1^{o} \text { and } 4^{o} \text { of Theorem }[6.4 \text { ) }\right. \\
& \left.=\widehat{\operatorname{RB}}_{\alpha+1}\left(R_{\mu}\right) \text { (by the definition of } \mathbb{B}_{\alpha+1}\right) \\
& =\widehat{\operatorname{RB}}_{\alpha}\left(\widehat{\operatorname{RB}}\left(R_{\mu}\right)\right) \text { (by Proposition }[5.9) \\
& =\widehat{\operatorname{RB}}_{\alpha}\left(B_{\mu}\right) .
\end{aligned}
$$

Yet another way of approaching the transformations $\mathbb{B}_{\alpha}$ can be obtained in terms of $\boxplus$ and $\uplus$ convolution powers.

Proposition 6.6. For every $\mu \in \Sigma_{\text {alg }}(\mathcal{B})$ and every linear transformation $\alpha: \mathcal{B} \rightarrow$ $\mathcal{B}$ one has that

$$
\left(\mathbb{B}_{\alpha}(\mu)\right)^{\uplus(1+\alpha)}=\mu^{\boxplus(1+\alpha)} .
$$


As a consequence, if $\alpha: \mathcal{B} \rightarrow \mathcal{B}$ is a linear transformation such that $1+\alpha$ is invertible, then the map $\mathbb{B}_{\alpha}: \Sigma_{\text {alg }}(\mathcal{B}) \rightarrow \Sigma_{\text {alg }}(\mathcal{B})$ can be described by the formula

$$
\mathbb{B}_{\alpha}(\mu)=\left(\mu^{\boxplus(1+\alpha)}\right)^{\uplus\left((1+\alpha)^{-1}\right)}, \quad \mu \in \Sigma_{\text {alg }}(\mathcal{B}) .
$$

Proof. We verify that the distributions on the two sides of (6.6) have the same $B$-transform. We start from the left-hand side:

$$
\begin{aligned}
B_{\left(\mathbb{B}_{\alpha}(\mu)\right)^{\uplus(1+\alpha)}} & =(1+\alpha) \circ B_{\left(\mathbb{B}_{\alpha}(\mu)\right)} \\
& =(1+\alpha) \circ \widehat{\mathrm{RB}}_{\alpha}\left(B_{\mu}\right) \text { (by Proposition 6.5) } \\
& =(1+\alpha) \circ \widehat{\mathrm{RB}}_{\alpha}\left(\widehat{\mathrm{RB}}\left(R_{\mu}\right)\right) \\
& =(1+\alpha) \circ \widehat{\mathrm{RB}}_{1+\alpha}\left(R_{\mu}\right) \\
& \left.=\widehat{\mathrm{RB}}\left((1+\alpha) \circ R_{\mu}\right) \text { (by Remark [5.6.3) } 3\right) \\
& =\widehat{\mathrm{RB}}\left(R_{\mu^{\boxplus(1+\alpha)}}\right) \\
& =B_{\mu^{\boxplus(1+\alpha)}} .
\end{aligned}
$$

We conclude this section by showing how the transformations $\mathbb{B}_{\alpha}$ relate to the $\mathcal{B}$ valued free Brownian motion. We do this by putting into evidence a transformation " $\Phi$ " which is the $\mathcal{B}$-valued analog for the transformation with the same role (and the same name) that was introduced in the $\mathbb{C}$-valued framework in 3 , 4. This operator-valued version of $\Phi$ is introduced in Definition 6.8 below, on a line similar to the one used in 4 in the multi-variable $\mathbb{C}$-valued framework. Before stating that, we briefly recall some basic terminology related to $\mathcal{B}$-valued semicircular elements and free Brownian motion.

Definition 6.7. For a linear map $\eta: \mathcal{B} \rightarrow \mathcal{B}$, we denote by $\gamma_{\eta}$ the distribution in $\Sigma_{a l g}(\mathcal{B})$ which is uniquely determined by the requirement that its $R$-transform acts as follows:

$$
R_{\gamma_{\eta}}^{[2]}=\eta, \text { and } R_{\gamma_{\eta}}^{[n]}=0 \text { for every } n \neq 2 .
$$

This $\gamma_{\eta}$ is called the $\mathcal{B}$-valued semicircular distribution of variance $\eta$. In the case when $\eta$ is completely positive, the distribution $\gamma_{\eta}$ belongs to $\Sigma^{0}(\mathcal{B})$ (see e.g. [15. 16]).

For $\mu \in \Sigma_{a l g}(\mathcal{B})$, the collection of distributions

$$
\left\{\mu \boxplus \gamma_{\eta} \mid \eta: \mathcal{B} \rightarrow \mathcal{B}, \text { linear }\right\}
$$

is sometimes referred to as the " $\mathcal{B}$-valued free Brownian motion started at $\mu$ ".

Definition 6.8. For $\beta: \mathcal{B}\langle\mathcal{X}\rangle \rightarrow \mathcal{B}$ a $\mathbb{C}$-linear map, define $\Phi[\beta] \in \Sigma_{\text {alg }}(\mathcal{B})$ by prescribing the $B$-transform of $\Phi[\beta]$ to act as follows:

$$
B_{\Phi[\beta]}^{[1]}=0 \in \mathcal{B}
$$

and then

$$
B_{\Phi[\beta]}^{[n]}\left[b_{1}, b_{2}, \ldots, b_{n-1}\right]=\beta\left[b_{1} \mathcal{X} \cdots \mathcal{X} b_{n-1}\right], \quad \forall n \geq 2, \forall b_{1}, \ldots, b_{n-1} \in \mathcal{B} .
$$


$\Phi$ is clearly a bijection:

$$
\begin{gathered}
\Phi:\{\mathbb{C} \text {-linear } \beta: \mathcal{B}\langle\mathcal{X}\rangle \rightarrow \mathcal{B}\} \rightarrow\{\text { unital } \mathcal{B} \text {-bimodule maps } \mu: \mathcal{B}\langle\mathcal{X}\rangle \rightarrow \mathcal{B} \\
\text { s.t. } \left.\left.\mu\right|_{\mathcal{B X} \mathcal{B}}=0\right\} .
\end{gathered}
$$

Note that if $\beta \in \Sigma_{a l g}(\mathcal{B})$, then $B_{\Phi[\beta]}^{[2]}\left[b_{1}\right]=b_{1}$ and for $n>2$,

$$
B_{\Phi[\beta]}^{[n]}\left[b_{1}, b_{2}, \ldots, b_{n-1}\right]=b_{1} M_{\beta}^{[n-2]}\left[b_{2}, \ldots, b_{n-2}\right] b_{n-1} .
$$

Theorem 6.9. Let $\alpha: \mathcal{B} \rightarrow \mathcal{B}$ be a linear transformation, $\beta \in \Sigma_{\text {alg }}(\mathcal{B})$, and $\left\{\gamma_{\alpha}\right\}$ be the $\mathcal{B}$-valued semicircular distribution with covariance $\alpha$. Then

$$
\Phi\left[\beta \boxplus \gamma_{\alpha}\right]=\mathbb{B}_{\alpha}[\Phi[\beta]] .
$$

Proof. We prove that the distributions on the two sides of (6.8) have the same $B$-transform. For $n=1$,

For $n \geq 2$,

$$
B_{\Phi\left[\beta \boxplus \gamma_{\alpha}\right]}^{[1]}=0=\widehat{\mathrm{RB}}_{\alpha}\left[B_{\Phi[\beta]}\right]^{[1]}=B_{\mathbb{B}_{\alpha}[\Phi[\beta]]}^{[1]} .
$$

$$
\begin{aligned}
B_{\Phi\left[\beta \boxplus \gamma_{\alpha}\right]}^{[n]}\left[b_{1}, b_{2}, \ldots, b_{n-1}\right] & b_{1} M_{\beta \boxplus \gamma_{\alpha}}^{[n-2]}\left(b_{2}, \ldots, b_{n-2}\right) b_{n-1} \\
& =\sum_{\pi \in N C(n-2)} \sum_{\substack{S \subset \pi \\
V \in S \Rightarrow|V|=2}} b_{1}\left(\alpha, R_{\beta}\right)^{\left[\pi, c_{\pi, S, 1}\right]}\left(b_{2}, \ldots, b_{n-2}\right) b_{n-1} \\
& =\sum_{\substack{\pi \ll 1_{n} \\
\left|V_{o}(\pi)\right|=2}} \sum_{\substack{V_{o}(\pi) \in S \subset \pi \\
V \in S \Rightarrow|V|=2}}\left(1, \alpha, R_{\beta}\right)^{\left[\pi, c_{\pi, S, 2}\right]}\left(b_{1}, b_{2}, \ldots, b_{n-2}, b_{n-1}\right),
\end{aligned}
$$

where

and

$$
c_{\pi, S, 1}= \begin{cases}1, & \text { if } V \in S, \\ 2, & \text { if } V \notin S,\end{cases}
$$

On the other hand,

$$
c_{\pi, S, 2}= \begin{cases}1, & \text { if } V=V_{o}(\pi), \\ 2, & \text { if } V \in S \text { but } V \neq V_{o}(\pi), \\ 3, & \text { if } V \notin S\end{cases}
$$

$$
\begin{aligned}
B_{\mathbb{B}_{\alpha}[\Phi[\beta]]}^{[n]}\left[b_{1}, b_{2}, \ldots, b_{n-1}\right] & =\widehat{\mathrm{RB}}_{\alpha}\left(B_{\Phi[\beta]}\right)^{[n]}\left[b_{1}, b_{2}, \ldots, b_{n-1}\right] \\
& =\sum_{\rho \ll 1_{n}}\left(B_{\Phi[\beta]}, \alpha \circ B_{\Phi[\beta]}\right)^{\left[\rho, \mathrm{O}_{\rho}\right]}\left[b_{1}, b_{2}, \ldots, b_{n-1}\right] .
\end{aligned}
$$

Note that $B_{\Phi[\beta]}^{[1]}=0$, so the sum above can be restricted to those $\rho$ each of whose classes contains at least 2 elements.

Given $\pi$ and $S=\left\{V_{1}, \ldots, V_{k}\right\}$ as above, we define a partition

$$
f(\pi, S)=\rho=\left(U_{1}, \ldots, U_{k}\right) \ll 1_{n}
$$

as follows: $V_{i} \subset U_{i}$, and $a \in U_{i}$ if for some $b, c \in V_{i}, b \leq a \leq c$, and for any $j \neq i$ and $d, e \in V_{j}, d \leq a \leq e$ implies $d<b \leq c<e$. Conversely, given $\rho=\left(U_{1}, \ldots, U_{k}\right)$ each of whose classes contains at least 2 elements, $V_{i}=\left\{\min \left(U_{i}\right), \max \left(U_{i}\right)\right\}$, and

$$
\pi=\widehat{\pi_{1}} \cup \cdots \cup \widehat{\pi_{k}}
$$


(in the notation of Lemma 3.5), where $\widehat{\pi}_{i} \ll 1_{U_{i}},\left|V_{o}\left(\widehat{\pi}_{i}\right)\right|=2$. See also Proposition 5.4, Theorem 6.2 and Remark 6.3 of [4], or Theorem 11 of [1]. To finish the proof, it suffices to show that for each $\rho \ll 1_{n}$,

$$
\begin{aligned}
& \left(B_{\Phi[\beta]}, \alpha \circ B_{\Phi[\beta]}\right)^{\left[\rho, \mathrm{O}_{\rho}\right]}\left[b_{1}, b_{2}, \ldots, b_{n-1}\right] \\
& =\sum_{(\pi, S) \in f^{-1}(\rho)}\left(1, \alpha, R_{\beta}\right)^{\left[\pi, c_{\pi, S, 2}\right]}\left(b_{1}, b_{2}, \ldots, b_{n-2}, b_{n-1}\right) \\
& =\sum_{\substack{\pi=\widehat{\pi_{1}} \cup \ldots \cup \widehat{\pi_{i}} \\
\pi_{i} \ll 1_{U_{i}} \\
\left|V_{o}\left(\pi_{i}\right)\right|=2}}\left(1, \alpha, R_{\beta}\right)^{\left[\pi, c_{\pi, S, 2}\right]}\left(b_{1}, b_{2}, \ldots, b_{n-2}, b_{n-1}\right) .
\end{aligned}
$$

Since, subject to these conditions, the $\left\{\widehat{\pi}_{i}\right\}$ can be chosen independently, it suffices to prove this equality for each class $U_{i} \in \rho$ separately. If $U_{i} \neq V_{o}(\rho)$, the expression is

$$
\begin{aligned}
\left(\alpha \circ B_{\Phi[\beta]}\right)^{\left[\left|U_{i}\right|\right]}\left[b_{1}, b_{2}, \ldots, b_{\left|U_{i}\right|-1}\right] & =\alpha\left[\beta\left[b_{1} \mathcal{X} b_{2} \mathcal{X} \ldots \mathcal{X} b_{\left|U_{i}\right|-1}\right]\right. \\
& =\sum_{\substack{\widehat{\pi}_{i} \ll 1_{U_{i}} \\
\left|V_{o}\left(\widehat{\pi}_{i}\right)\right|=2}}\left(\alpha, R_{\beta}\right)^{\left[\pi, \mathrm{O}_{\widehat{\pi}_{i}}\right]}\left(b_{1}, b_{2}, \ldots, b_{n-2}, b_{\left|U_{i}\right|-1}\right),
\end{aligned}
$$

while for $U_{i}=V_{o}(\rho)$ the expression is the same without $\alpha$. The result follows.

\section{Operator MODELS}

In the remainder of the paper, we will denote by $\mathcal{C P}(\mathcal{B})$ the space of completely positive $\mathbb{C}$-linear maps on $\mathcal{B}$.

Construction 7.1. Let $\lambda \in \mathcal{B}$ be symmetric, and $\beta: \mathcal{B}\langle\mathcal{X}\rangle \rightarrow \mathcal{B}$ be a $\mathbb{C}$-linear, completely positive map. Define the $\mathcal{B}$-valued inner product on $\mathcal{B}_{0}\langle\mathcal{X}\rangle$ by

$$
\left\langle b_{0} \mathcal{X} \ldots \mathcal{X} b_{n}, c_{0} \mathcal{X} \ldots \mathcal{X} c_{k}\right\rangle_{\beta}=c_{k}^{*} \beta\left[c_{k-1}^{*} \mathcal{X} \ldots \mathcal{X} c_{0}^{*} b_{0} \mathcal{X} \ldots \mathcal{X} b_{n-1}\right] b_{n} .
$$

Note that a general element of $\mathcal{B}_{0}\langle\mathcal{X}\rangle$ is of the form $\sum_{i=1}^{n} P_{i} \mathcal{X} b_{i}$, with $P_{i} \in \mathcal{B}\langle\mathcal{X}\rangle$, and

$$
\left\langle\sum_{i=1}^{n} P_{i} \mathcal{X} b_{i}, \sum_{i=1}^{n} P_{i} \mathcal{X} b_{i}\right\rangle_{\beta}=\sum_{i, j=1}^{n} b_{i}^{*} \beta\left[P_{i}^{*} P_{j}\right] b_{j} \geq 0
$$

since $\beta$ is completely positive. It follows that this inner product is positive. It may be degenerate; however, we will only use this construction for combinatorial computations of moments and cumulants.

Lemma 7.2. Consider the vector space $\mathcal{B}\langle\mathcal{X}\rangle=\mathcal{B} \oplus \mathcal{B}_{0}\langle\mathcal{X}\rangle$ with the $\mathcal{B}$-valued inner product

$$
\left\langle b \oplus m, b^{\prime} \oplus m^{\prime}\right\rangle=\left(b^{\prime}\right)^{*} b+\left\langle m, m^{\prime}\right\rangle_{\beta} .
$$

On this vector space, we define maps

$$
\begin{gathered}
a^{*}\left(b \oplus b_{0} \mathcal{X} \ldots \mathcal{X} b_{n}\right)=0 \oplus \mathcal{X} b \\
p\left(b \oplus b_{0} \mathcal{X} \ldots \mathcal{X} b_{n}\right)=0 \oplus \mathcal{X} b_{0} \mathcal{X} \ldots \mathcal{X} b_{n},
\end{gathered}
$$

and

$$
a\left(b \oplus b_{0} \mathcal{X} \ldots \mathcal{X} b_{n}\right)=\beta\left[b_{0} \mathcal{X} \ldots \mathcal{X} b_{n-1}\right] b_{n} \oplus 0
$$

in particular,

$$
a\left(b \oplus b_{0} \mathcal{X}\right)=\beta\left[b_{0}\right] \oplus 0 .
$$


Then $p$ and $a^{*}+a$ are symmetric. Therefore the operator

$$
X=a^{*}+a+p+\lambda
$$

is also symmetric. It follows that

$$
\mu_{(\lambda, \beta)}: \mathcal{B}\langle\mathcal{X}\rangle \rightarrow \mathcal{B}
$$

defined via

$$
\mu_{(\lambda, \beta)}\left[b_{0} \mathcal{X} \ldots \mathcal{X} b_{n}\right]=\left\langle\left(b_{0} X \ldots X b_{n}\right)(1 \oplus 0), 1 \oplus 0\right\rangle,
$$

is a conditional expectation, $\mu_{(\lambda, \beta)} \in \Sigma(\mathcal{B})$. Moreover, if $\beta$ satisfies the boundedness condition (2.8), then $\mu_{(\lambda, \beta)} \in \Sigma^{0}(\mathcal{B})$.

Proof. We check the first statement. Indeed,

$$
\begin{aligned}
\left\langle a^{*}\left(b \oplus b_{0} \mathcal{X} \ldots \mathcal{X} b_{n}\right), c \oplus c_{0} \mathcal{X} \ldots \mathcal{X} c_{k}\right\rangle & =\left\langle 0 \oplus \mathcal{X} b, c \oplus c_{0} \mathcal{X} \ldots \mathcal{X} c_{k}\right\rangle \\
& =c_{k}^{*} \beta\left[c_{k-1}^{*} \mathcal{X} \ldots \mathcal{X} c_{0}^{*}\right] b \\
& =\left\langle b \oplus 0, a\left(c \oplus c_{0} \mathcal{X} \ldots \mathcal{X} c_{k}\right)\right\rangle \\
& =\left\langle b \oplus b_{0} \mathcal{X} \ldots \mathcal{X} b_{n}, a\left(c \oplus c_{0} \mathcal{X} \ldots \mathcal{X} c_{k}\right)\right\rangle
\end{aligned}
$$

and

$$
\begin{aligned}
\left\langle p\left(b \oplus b_{0} \mathcal{X} \ldots \mathcal{X} b_{n}\right), c \oplus c_{0} \mathcal{X} \ldots \mathcal{X} c_{k}\right\rangle & =\left\langle 0 \oplus \mathcal{X} b_{0} \mathcal{X} \ldots \mathcal{X} b_{n}, c \oplus c_{0} \mathcal{X} \ldots \mathcal{X} c_{k}\right\rangle \\
& =c_{k}^{*} \beta\left[c_{k-1}^{*} \mathcal{X} \ldots \mathcal{X} c_{0}^{*} \mathcal{X} b_{0} \mathcal{X} \ldots \mathcal{X} b_{n-1}\right] b_{n} \\
& =\left\langle b \oplus b_{0} \mathcal{X} \ldots \mathcal{X} b_{n}, 0 \oplus \mathcal{X} c_{0} \mathcal{X} \ldots \mathcal{X} c_{k}\right\rangle \\
& =\left\langle b \oplus b_{0} \mathcal{X} \ldots \mathcal{X} b_{n}, p\left(c \oplus c_{0} \mathcal{X} \ldots \mathcal{X} c_{k}\right)\right\rangle .
\end{aligned}
$$

It follows that $\mu_{(\lambda, \beta)} \in \Sigma(\mathcal{B})$. Finally, if $\beta$ satisfies (2.8) with constant $M$ and $\|\lambda\| \leq M$, then from (7.2) below,

$$
\left\|\mu_{(\lambda, \beta)}\left[\mathcal{X} b_{1} \mathcal{X} \ldots b_{n-1} \mathcal{X}\right]\right\| \leq 2^{n} M^{n}\left\|b_{1}\right\| \cdot\left\|b_{2}\right\| \cdot \ldots \cdot\left\|b_{n-1}\right\| .
$$

Lemma 7.3. The Boolean cumulant functionals $B_{(\lambda, \beta)}$ of $\mu_{(\lambda, \beta)}$ are

$$
B_{(\lambda, \beta)}^{[1]}=\lambda
$$

and

$$
B_{(\lambda, \beta)}^{[n]}\left(b_{1}, b_{2}, \ldots, b_{n-1}\right)=\beta\left[b_{1} \mathcal{X} \ldots \mathcal{X} b_{n-1}\right] .
$$

Proof. Using the definition $X=a^{*}+a+p+\lambda$ and the definitions of $a^{*}, a, p, \lambda$,

$$
\begin{aligned}
& \mu_{(\lambda, \beta)}\left[\mathcal{X} b_{1} \ldots b_{n-1} \mathcal{X}\right]=\left\langle\left(X b_{1} \ldots b_{n-1} X\right)(1 \oplus 0), 1 \oplus 0\right\rangle \\
&= \sum_{k=1}^{n} \sum_{1 \leq i_{1}<i_{2}<\ldots<i_{k}=n} \beta\left[b_{1} \mathcal{X} \ldots b_{i_{1}-1}\right] b_{i_{1}} \beta\left[b_{i_{1}+1} \mathcal{X} \ldots b_{i_{2}-1}\right] b_{i_{2}} \\
& \ldots b_{i_{k-1}} \beta\left[b_{i_{k-1}+1} \mathcal{X} \ldots b_{n-1}\right],
\end{aligned}
$$

where $\beta[\emptyset]=\lambda$. On the other hand, combining the last formula in Remark 3.7. Notation 4.4, and Definition 4.6, we get

$$
\begin{gathered}
M_{\mu}^{[n]}\left(b_{1}, \ldots, b_{n-1}\right)=\sum_{k=1}^{n} \sum_{1 \leq i_{1}<i_{2}<\ldots<i_{k}=n} B_{\mu}\left(b_{1}, \ldots, b_{i_{1}-1}\right) b_{i_{1}} B_{\mu}\left(b_{i_{1}+1}, \ldots, b_{i_{2}-1}\right) b_{i_{2}} \\
\ldots b_{i_{k-1}} B_{\mu}\left(b_{i_{k-1}+1}, \ldots, b_{n-1}\right) .
\end{gathered}
$$

Comparing these two formulas, we get the result. 
Compare with Theorem 5.6 of 13 .

The following result follows from Lemma 2.9 and Theorem 2.5 of [13, but for completeness we provide a shorter proof for our case.

Lemma 7.4. Let $\mu \in \Sigma(\mathcal{B})$. Then for some symmetric element $\lambda \in \mathcal{B}$ and $a$ $\mathbb{C}$-linear, completely positive map $\beta: \mathcal{B}\langle\mathcal{X}\rangle \rightarrow \mathcal{B}$, we have

$$
\mu=\mu_{(\lambda, \beta)} .
$$

If $\mu \in \Sigma^{0}(\mathcal{B})$, then $\beta$ satisfies condition (2.8).

Proof. Put on $\mathcal{B}\langle\mathcal{X}\rangle$ the $\mathcal{B}$-valued inner product

$$
\left\langle b_{0} \mathcal{X} \ldots \mathcal{X} b_{n}, c_{0} \mathcal{X} \ldots \mathcal{X} c_{k}\right\rangle_{\mu}=\mu\left[c_{k}^{*} \mathcal{X} c_{k-1}^{*} \mathcal{X} \ldots \mathcal{X} c_{0}^{*} b_{0} \mathcal{X} \ldots \mathcal{X} b_{n-1} \mathcal{X} b_{n}\right],
$$

and denote by $\mathcal{H}$ the corresponding $\mathcal{B}$-inner product bimodule. We will identify elements of $\mathcal{B}\langle\mathcal{X}\rangle$ with corresponding operators acting on $\mathcal{H}$ on the left. Denote by

$$
P: \zeta \mapsto \mu[\zeta]
$$

the orthogonal projection from $\mathcal{H}$ onto $\mathcal{B} \subset \mathcal{H}$, and let $P^{\perp}=I-P$, so that

$$
P^{\perp}: \zeta \mapsto \zeta-\mu[\zeta]
$$

We write

$$
\xi=P^{\perp} \mathcal{X} \cdot 1=\mathcal{X}-\mu[\mathcal{X}] \in \mathcal{H}
$$

Finally, denote $T=P^{\perp} \mathcal{X} P^{\perp}$; clearly $T$ is a symmetric operator on $\mathcal{H}$.

Now using the fact that $P$ and $P^{\perp}$ commute with $\mathcal{B}$ (in their actions on $\mathcal{H}$ ), and

$$
\langle\chi P \zeta \cdot 1,1\rangle_{\mu}=\mu[\chi] \mu[\zeta],
$$

we compute

$$
\begin{aligned}
\mu\left[b_{0} \mathcal{X} b_{1} \ldots\right. & \left.b_{n-1} \mathcal{X} b_{n}\right] \\
= & b_{0}\left\langle\mathcal{X} b_{1} \ldots b_{n-1} \mathcal{X} \cdot 1,1\right\rangle_{\mu} b_{n} \\
= & b_{0}\left\langle\mathcal{X} b_{1}\left(P+P^{\perp}\right) \mathcal{X} b_{2} \ldots b_{n-2}\left(P+P^{\perp}\right) \mathcal{X} b_{n-1}\left(P+P^{\perp}\right) \mathcal{X} \cdot 1,1\right\rangle_{\mu} b_{n} \\
= & \sum_{k=1}^{n} \sum_{1 \leq i_{1}<i_{2}<\ldots<i_{k}=n} b_{0}\left\langle\mathcal{X} b_{1} P^{\perp} \mathcal{X} \ldots P^{\perp} \mathcal{X} b_{i_{1}-1} P^{\perp} \mathcal{X} \cdot 1,1\right\rangle_{\mu} b_{i_{1}} \\
& \quad \ldots b_{i_{k-1}}\left\langle\mathcal{X} b_{i_{k-1}+1} P^{\perp} \mathcal{X} \ldots P^{\perp} \mathcal{X} b_{n-1} P^{\perp} \mathcal{X} \cdot 1,1\right\rangle_{\mu} b_{n} \\
= & \sum_{k=1}^{n} \sum_{1 \leq i_{1}<i_{2}<\ldots<i_{k}=n} b_{0}\left\langle b_{1} T b_{2} \ldots T b_{i_{1}-1} \xi, \xi\right\rangle_{\mu} b_{i_{1}} \\
& \ldots b_{i_{k-1}}\left\langle b_{i_{k-1}+1} T \ldots T b_{n-1} \xi, \xi\right\rangle_{\mu} b_{n} .
\end{aligned}
$$

Note that we have used the bimodule property of the $\mu$-inner product. Comparing with formula (7.2), we see that $\mu=\mu_{(\lambda, \beta)}$ for

$$
\lambda=\langle\mathcal{X} \cdot 1,1\rangle_{\mu}=\mu[\mathcal{X}]
$$

and

$$
\beta\left[b_{1} \mathcal{X} b_{2} \ldots \mathcal{X} b_{n}\right]=\left\langle b_{1} T b_{2} \ldots T b_{n} \xi, \xi\right\rangle_{\mu}
$$


Clearly $\lambda$ is symmetric, and since $T$ is symmetric, $\beta$ is positive. In fact,

$$
\begin{aligned}
\sum_{i, j=1}^{n} & c_{i}^{*} \beta\left[\left(b_{i, 1} \mathcal{X} b_{i, 2} \ldots \mathcal{X} b_{i, k(i)}\right)^{*}\left(b_{j, 1} \mathcal{X} b_{j, 2} \ldots \mathcal{X} b_{j, k(j)}\right)\right] c_{j} \\
& =\sum_{i, j=1}^{n}\left\langle\left(b_{i, 1} T b_{i, 2} \ldots T b_{i, k(i)}\right)^{*}\left(b_{j, 1} T b_{j, 2} \ldots T b_{j, k(j)}\right) \xi c_{j}, \xi c_{i}\right\rangle_{\mu} \\
& =\left\langle\sum_{i=1}^{n}\left(b_{i, 1} T b_{i, 2} \ldots T b_{i, k(i)}\right) \xi c_{i}, \sum_{i=1}^{n}\left(b_{i, 1} T b_{i, 2} \ldots T b_{i, k(i)}\right) \xi c_{i}\right\rangle_{\mu} \geq 0
\end{aligned}
$$

so $\beta$ is completely positive. If $\mu \in \Sigma^{0}(\mathcal{B})$, then, since $\|T\|=\left\|P^{\perp} \mathcal{X} P^{\perp}\right\| \leq\|\mathcal{X}\|, \beta$ satisfies condition (2.8).

Theorem 7.5. For any $\mu \in \Sigma(\mathcal{B})$ and $\alpha \in \mathcal{C P}(\mathcal{B})$, the functional $\mu^{\uplus \alpha} \in \Sigma(\mathcal{B})$. Moreover, if $\mu \in \Sigma^{0}(\mathcal{B})$, so is $\mu^{\uplus \alpha}$.

Proof. By the preceding lemma, $\mu=\mu_{(\lambda, \beta)}$ for some $(\lambda, \beta)$. Moreover, replacing the pair $(\lambda, \beta)$ with the pair $(\alpha[\lambda], \alpha \circ \beta)$ gives another pair of the same type, so

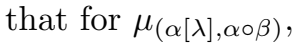

$$
B_{(\alpha[\lambda], \alpha \circ \beta)}^{[n]}\left(b_{1}, b_{2}, \ldots, b_{n-1}\right)=\alpha\left[B_{(\lambda, \beta)}^{[n]}\left(b_{1}, b_{2}, \ldots, b_{n-1}\right)\right]
$$

for any $n \geq 1$. So by Definition 4.7

$$
\mu_{(\alpha[\lambda], \alpha \circ \beta)}=\mu_{(\lambda, \beta)}^{\uplus \alpha} .
$$

The statement about $\Sigma^{0}(\mathcal{B})$ also follows from the preceding lemmas.

Corollary 7.6. For any freely infinitely divisible $\mu \in \Sigma(\mathcal{B})$ and any $\alpha \in \mathcal{C P}(\mathcal{B})$, the functional $\mu^{\boxplus \alpha} \in \Sigma(\mathcal{B})$. Moreover, if $\mu \in \Sigma^{0}(\mathcal{B})$, so is $\mu^{\boxplus \alpha}$.

Proof. Theorem 3.4 of [7] shows that the Bercovici-Pata bijection $\mathbb{B}$ from Definition 6.1 maps $\Sigma(\mathcal{B})$ bijectively onto freely infinitely divisible elements of $\Sigma(\mathcal{B})$, $\Sigma^{0}(\mathcal{B})$ bijectively onto freely infinitely divisible elements of $\Sigma^{0}(\mathcal{B})$, and intertwines $\uplus$ and $\boxplus$. So the result follows from the preceding proposition.

Remark 7.7. The preceding corollary can also be proved directly. The full Fock module over $\mathcal{B}_{0}\langle\mathcal{X}\rangle$ is the $\mathcal{B}$-bimodule

$$
\mathcal{F}\left(\mathcal{B}_{0}\langle\mathcal{X}\rangle\right)=\bigoplus_{k=0}^{\infty} \mathcal{B}_{0}\langle\mathcal{X}\rangle^{\otimes_{\mathcal{B}}^{k}}=\mathcal{B} \oplus \mathcal{B}_{0}\langle\mathcal{X}\rangle \oplus\left(\mathcal{B}_{0}\langle\mathcal{X}\rangle \otimes_{\mathcal{B}} \mathcal{B}_{0}\langle\mathcal{X}\rangle\right) \oplus \ldots
$$

Given $\lambda$ and $\beta$ as in Construction 7.1 , on $\mathcal{F}\left(\mathcal{B}_{0}\langle\mathcal{X}\rangle\right)$, define the $\mathcal{B}$-valued inner product by

$$
\left\langle\xi_{1} \otimes \ldots \otimes \xi_{k}, \zeta_{1} \otimes \ldots \otimes \zeta_{n}\right\rangle=\delta_{k n}\left\langle\xi_{n},\left\langle\xi_{n-1}, \ldots\left\langle\xi_{1}, \zeta_{1}\right\rangle_{\beta} \ldots \zeta_{n-1}\right\rangle_{\beta} \zeta_{n}\right\rangle_{\beta},
$$

where $\langle\cdot, \cdot\rangle_{\beta}$ is given in formula (7.1). Again, positivity of the inner product follows from complete positivity of $\beta$; compare with Definition 4.6.5 from [16. On $\mathcal{F}\left(\mathcal{B}_{0}\langle\mathcal{X}\rangle\right)$, we define maps

$$
\begin{gathered}
a^{*}\left(\xi_{1} \otimes \ldots \otimes \xi_{n}\right)=\mathcal{X} \otimes \xi_{1} \otimes \ldots \otimes \xi_{n}, \\
p\left(\xi_{1} \otimes \ldots \otimes \xi_{n}\right)=\mathcal{X} \xi_{1} \otimes \ldots \otimes \xi_{n},
\end{gathered}
$$


and

$$
a\left(\xi_{1} \otimes \ldots \otimes \xi_{n}\right)=\left\langle\xi_{1}, \mathcal{X}\right\rangle_{\beta} \xi_{2} \otimes \ldots \otimes \xi_{n} .
$$

Then as in Lemma 7.2, $p, a^{*}+a$, and

$$
X=a^{*}+a+p+\lambda
$$

are symmetric, and the functional $\mu$ defined via

$$
\mu\left[b_{0} \mathcal{X} \ldots \mathcal{X} b_{n}\right]=\left\langle\left(b_{0} X \ldots X b_{n}\right) 1,1\right\rangle
$$

is a conditional expectation. By definition (cf. Section 4.7 of [16] or Section 3 of [13]), the conditional expectations arising in this construction are precisely all the freely infinitely divisible ones. Moreover, this $\mu=\mathbb{B}\left[\mu_{(\lambda, \beta)}\right]$, and the conditional expectation arising from $(\alpha[\lambda], \alpha \circ \beta)$ is $\mu^{\boxplus \alpha}$.

Theorem 7.8. For each $\alpha \in \mathcal{C P}(\mathcal{B}), \mathbb{B}_{\alpha}$ maps $\Sigma(\mathcal{B})$ to itself.

Proof. Let $\mu \in \Sigma(\mathcal{B})$, so that $\mu=\mu_{(\lambda, \beta)}$ for some $\lambda, \beta$ as in Construction7.1. Modify the construction in Remark 7.7 as follows. On $\mathcal{F}\left(\mathcal{B}_{0}\langle\mathcal{X}\rangle\right)$, define the $\mathcal{B}$-valued inner product by

$$
\left\langle\xi_{1} \otimes \ldots \otimes \xi_{k}, \zeta_{1} \otimes \ldots \otimes \zeta_{n}\right\rangle=\delta_{k n}\left\langle\xi_{n},\left\langle\xi_{n-1}, \ldots\left\langle\xi_{1}, \zeta_{1}\right\rangle_{\alpha \circ \beta} \ldots \zeta_{n-1}\right\rangle_{\alpha \circ \beta} \zeta_{n}\right\rangle_{\beta} ;
$$

in particular,

$$
\langle\xi, \zeta\rangle=\langle\xi, \zeta\rangle_{\beta}
$$

Keep $a^{*}, p$, and $a(\xi)$ the same, and let

$$
a\left(\xi_{1} \otimes \ldots \otimes \xi_{n}\right)=\left\langle\xi_{1}, \mathcal{X}\right\rangle_{\alpha \circ \beta} \xi_{2} \otimes \ldots \otimes \xi_{n}
$$

for $n \geq 2$. Also, let $L(\xi)=\lambda \xi$ while $L\left(\xi_{1} \otimes \ldots \otimes \xi_{n}\right)=\alpha[\lambda] \xi_{1} \otimes \ldots \otimes \xi_{n}$ for $n \geq 2$. Then again, $X=a^{*}+a+p+L$ is symmetric, and the functional $\mu_{\alpha}$ defined via

$$
\mu_{\alpha}\left[b_{0} \mathcal{X} \ldots \mathcal{X} b_{n}\right]=\left\langle\left(b_{0} X \ldots X b_{n}\right) 1,1\right\rangle
$$

is in $\Sigma(\mathcal{B})$. But now it is easy to check that $B_{\mu_{\alpha}}=\widehat{\mathrm{RB}}_{\alpha}\left(B_{\mu}\right)$, which implies that $\mu_{\alpha}=\mathbb{B}_{\alpha}(\mu)$.

Theorem 7.9. Let $\mu \in \Sigma(\mathcal{B})$ and $\alpha \in \mathcal{C P}(\mathcal{B})$ such that $\alpha-1$ is completely positive. Then the functional $\mu^{\boxplus \alpha} \in \Sigma(\mathcal{B})$. If $\mu \in \Sigma^{0}(\mathcal{B})$, so is $\mu^{\boxplus \alpha}$.

Proof. The left-hand side of the formula (6.6) in Proposition 6.6 is well defined and positive by the preceding proposition; therefore so is the right-hand side. The boundedness also follows.

Remark 7.10. Note that we assume $\mu[1]=1$, and $\mu$ is a $\mathcal{B}$-bimodule map, so the restriction $\left.\mu\right|_{\mathcal{B}}$ is the identity map, and $\mu$ is an analog of a probability measure. On the other hand, $\beta$ is not necessarily a $\mathcal{B}$-bimodule map, and the restriction $\left.\beta\right|_{\mathcal{B}}$ is a general completely positive map. So $\beta$ is an analog of a general finite measure.

Corollary 7.11. For completely positive $\beta$,

$$
\Phi[\beta]=\mu_{(0, \beta)} .
$$

So $\Phi$ is also a bijection

$\Phi:\{\mathbb{C}$-linear, completely positive $\beta: \mathcal{B}\langle\mathcal{X}\rangle \rightarrow \mathcal{B}\} \rightarrow\left\{\mu \in \Sigma(\mathcal{B})\right.$ s.t. $\left.\left.\mu\right|_{\mathcal{B X} \mathcal{B}}=0\right\}$ 
with a restriction to $\beta$ satisfying (2.8) and $\mu \in \Sigma^{0}(\mathcal{B})$. It also restricts to a bijection

$$
\begin{aligned}
\Phi:\left\{\mathbb{C} \text {-linear, completely positive } \beta: \mathcal{B}\langle\mathcal{X}\rangle \rightarrow \mathcal{B} \text { s.t. }\left.\beta\right|_{\mathcal{B}}=I\right\} \\
\rightarrow\left\{\mu \in \Sigma(\mathcal{B}) \text { s.t. }\left.\mu\right|_{\mathcal{B X} \mathcal{B}}=0, \mu[\mathcal{X} b \mathcal{X}]=b\right\}
\end{aligned}
$$

Remark 7.12. For $\beta$ completely positive but not necessarily a $\mathcal{B}$-bimodule or a unital map, $\mathbb{B}_{\alpha}[\Phi[\beta]] \in \Sigma(\mathcal{B})$ and is centered, so by the preceding corollary it is in the image of $\Phi$. Therefore following Theorem 6.9, one can define

$$
\beta \mapsto \beta \boxplus \gamma_{\alpha}=\left(\Phi^{-1} \circ \mathbb{B}_{\alpha} \circ \Phi\right)[\beta],
$$

and this transformation preserved complete positivity. One can define the same extension of the free convolution operation using combinatorics, but in that case positivity is unclear.

\section{Analytic aspects: Analytic subordination and the OPERATOR-VALUED INVISCID BURGERS EQUATION}

This section is dedicated to a brief outline of some analytic consequences and aspects of our previous results.

8.1. The operator-valued analogue of the free heat equation. One of the fundamental results of Voiculescu was finding the free analogue of the heat equation. If $X=X^{*}$ is free from the centered semicircular random variable $S$ of variance one, then

where

$$
\frac{\partial G(t, z)}{\partial t}+G(t, z) \frac{\partial G(t, z)}{\partial z}=0, \quad \Im z>0, t>0
$$

$$
G(t, z)=G_{X+\sqrt{t} S}(z)=\int_{\mathbb{R}} \frac{1}{z-x} d \mu_{X+\sqrt{t} S} .
$$

We shall naturally extend this to the case when $X$ and $S$ are free over $\mathcal{B}$, the $\mathcal{B}$ valued centered semicircular random variable $S$ has variance $\eta$ and its evolution is as before according to (completely) positive maps $\rho: \mathcal{B} \rightarrow \mathcal{B}$.

We shall consider maps $\mathcal{C} \mathcal{P}(\mathcal{B}) \times \mathcal{B} \rightarrow \mathcal{B}$ analytic on some open set in the second coordinate, and Gâteaux differerentiable in the first.

Proposition 8.1. Assume that the map hatisfies

$$
h(\eta, b)=h_{0}(b+\eta(h(\eta, b))), \quad \eta \in \mathcal{C P}(\mathcal{B}), \Im b>0 .
$$

If $h_{0}$ is analytic on the set $\{b \in \mathcal{B}: \Im b>0\}$ and $h(\eta, b): \mathcal{C P}(\mathcal{B}) \times\{b \in \mathcal{B}: \Im b>$ $0\} \rightarrow\{b \in \mathcal{B}: \Im b>0\}$, then the following equation is satisfied:

$$
\frac{\partial h(\eta, b)}{\partial \eta}(\rho)-\frac{\partial h(\eta, b)}{\partial b}(\rho(h(\eta, b)))=0
$$

where $\rho \in \mathcal{C P}(\mathcal{B}), \eta \in \operatorname{Int}(\mathcal{C P}(\mathcal{B}))$, and $\Im b>0$. The derivative with respect to $b$ is in the Fréchet sense, and the derivative with respect to $\eta$ is taken in the Gâteaux sense.

Proof. The proof simply consists in applying the corresponding definitions and the "chain rule". Let $\eta$ and $\rho$ be as above. Strictly for convenience, we shall write $\omega=\omega(\eta, b)=b+\eta(h(\eta, b))$ and express the above in terms of $\omega$ as

$$
\omega(\eta, b)=b+\eta\left(h_{0}(\omega(\eta, b))\right), \quad \eta \in \mathcal{C P}(\mathcal{B}), \Im b>0 .
$$


Then

$$
\lim _{t \rightarrow 0} \frac{\omega(\eta+t \rho, b)-\omega(\eta, b)}{t}=\lim _{t \rightarrow 0} \frac{(\eta+t \rho)\left(h_{0}(\omega(\eta+t \rho, b))\right)-\eta\left(h_{0}(\omega(\eta, b))\right)}{t} .
$$

The right-hand side is easily seen to be equal to

$$
\rho\left(h_{0}(\omega(\eta, b))\right)+\left(\eta \circ h_{0}^{\prime}(\omega(\eta, b))\right)\left(\lim _{t \rightarrow 0} \frac{\omega(\eta+t \rho, b)-\omega(\eta, b)}{t}\right) .
$$

Fréchet differentiating in the variable $b$, we obtain

$$
\frac{\partial \omega(\eta, b)}{\partial b}=\operatorname{Id}_{\mathcal{B}}+\eta \circ h_{0}^{\prime}(\omega(\eta, b)) \circ \frac{\partial \omega(\eta, b)}{\partial b},
$$

where the above is an equality of linear endomorphisms of $\mathcal{B}$. From (8.2) and (8.3) we easily obtain

$$
\lim _{t \rightarrow 0} \frac{\omega(\eta+t \rho, b)-\omega(\eta, b)}{t}=\left(\operatorname{Id}_{\mathcal{B}}-\eta \circ h_{0}^{\prime}(\omega(\eta, b))\right)^{-1}\left(\rho\left(h_{0}(\omega(\eta, b))\right)\right),
$$

while (8.4) assures us that the linear operator $\operatorname{Id}_{\mathcal{B}}-\eta \circ h_{0}^{\prime}(\omega(\eta, b))$ is indeed invertible, as

$$
\left(\operatorname{Id}_{\mathcal{B}}-\eta \circ h_{0}^{\prime}(\omega(\eta, b))\right) \circ \frac{\partial \omega(\eta, b)}{\partial b}=\operatorname{Id}_{\mathcal{B}}
$$

Combining (8.5) and (8.6) provides us with the differential equation satisfied by $\omega$, which is of interest in its own right:

$(8.7)$

$\frac{\partial \omega(\eta, b)}{\partial \eta}(\rho)=\frac{\partial \omega(\eta, b)}{\partial b}\left(\rho\left(h_{0}(\omega(\eta, b))\right)\right), \quad \Im b>0, \eta \in \mathcal{C P}(\mathcal{B})$ invertible, $\rho \in \mathcal{C P}(\mathcal{B})$.

We recall the definition of $\omega$ and note that $h(\eta, b)=h_{0}(\omega(\eta, b))$ in order to obtain

$$
\rho(h(\eta, b))+\eta\left(\frac{\partial h(\eta, b)}{\partial \eta}(\rho)\right)=\rho(h(\eta, b))+\left(\eta \circ \frac{\partial h(\eta, b)}{\partial b}\right)(\rho(h(\eta, b))),
$$

and we conclude by the invertibility of $\eta$.

Remark 8.2. Let us justify why maps satisfying the conditions of the above proposition are important for our paper.

A bit of review: denoting $G_{\mu}(b)=b^{-1}+b^{-1} M_{\mu}\left(b^{-1}\right) b^{-1}$ (recall (2.10) $)$, we observe that $G_{\mu}(b)=\mu\left[(b-\mathcal{X})^{-1}\right]$ is an extension of $G_{\mu}$ to elements $b \in \mathcal{B}, \Im b>$ 0 . As noted in [21], $G_{\mu}(b)$ is invertible in $\mathcal{B}$ whenever $\Im b>0$ and, moreover, $\Im\left(G_{\mu}(b)^{-1}\right) \geq \Im b$. We also record here the fact that, as shown in [20], one has

$$
\mu\left[\left(1+b R_{\mu}(b)-b \mathcal{X}\right)^{-1}\right]=1, \quad\|b\| \text { small. }
$$

Now, let us denote $h_{\mu}(b):=G_{\mu}(b)^{-1}-b$, $\Im b>0$. It follows easily from the definition of $B_{\mu}$ that $B_{\mu}(b)=-h_{\mu}\left(b^{-1}\right)$. Using the definition of the transformation $\mathbb{B}_{\alpha}$, we note that $h_{\mathbb{B}_{\alpha}(\mu)}(b)=(1+\alpha)^{-1} h_{\mu^{\boxplus(1+\alpha)}}(b)=h_{\mu}\left(b+\alpha(1+\alpha)^{-1} h_{\mu^{\boxplus(1+\alpha)}}(b)\right)=$ $h_{\mu}\left(b+\alpha h_{\mathbb{B}_{\alpha}(\mu)}(b)\right)$. (We have used (4.12) for the first equality, (4.11), (8.8), as well as analytic continuation for the second, and direct substitution from the first for the third equality.) Thus, taking $h(\eta, b)=h_{\mathbb{B}_{\eta}(\mu)}(b)$ provides us with an example of a map satisfying the conditions of the above proposition. 
Theorem 8.3. Assume that $X=X^{*}$ and that $S$ are free over $\mathcal{B}$ and that $S$ is a $\mathcal{B}$-valued centered semicircular random variable of invertible variance $\eta$. If we denote $G(\eta, b)=\mu_{X+S}\left[(b-\mathcal{X})^{-1}\right], \Im b>0$, then

$$
\frac{\partial G(\eta, b)}{\partial \eta}(\rho)+\frac{\partial G(\eta, b)}{\partial b}(\rho(G(\eta, b)))=0,
$$

where $\rho \in \mathcal{C P}(\mathcal{B}), \eta \in \operatorname{Int}(\mathcal{C P}(\mathcal{B}))$, and $\Im b>0$. The derivative with respect to $b$ is in the Fréchet sense, and the derivative with respect to $\eta$ is taken in the Gâteaux sense.

Proof. This is an immediate consequence of Proposition 8.1, Theorem 6.9 and the above remarks. Indeed, as we know that the $R$-transform of $S$ is $R_{\mu_{S}}(b)=\eta(b)$, it follows that

$$
R_{\mu_{S+X}}(b)=R_{\mu_{X}}(b)+R_{\mu_{S}}(b)=R_{\mu_{X}}(b)+\eta(b), \quad\|b\| \text { small. }
$$

After adding $b^{-1}$ to both sides of the above equation, the definition of the $R$ transform in terms of $G_{\mu_{X}}(b)=\mu_{X}\left[(b-\mathcal{X})^{-1}\right]$ allows us to rewrite it as $b=$ $\eta\left(G_{\mu_{X+S}}(b)\right)+G_{\mu_{X}}^{-1}\left(G_{\mu_{X+S}}(b)\right)$. This equation holds when $\Im b>0$ and $\left\|b^{-1}\right\|$ is small enough. Moving $\eta\left(G_{\mu_{X+S}}(b)\right)$ to the left, composing with $G_{\mu_{X}}$ on the left and applying analytic continuation allows us to find the condition of Proposition 8.1 satisfied by $h_{0}=-G_{\mu_{X}}$ and $h(\eta, b)=-G_{\mu_{X+S}}(b)$, for all $b$ with strictly positive imaginary part.

8.2. Analytic subordination for $G_{\mu_{X}^{\boxplus \alpha}}$. Given $\mu \in \Sigma(\mathbb{C})$, an observation important in the study of the semigroup $\left\{\mu^{\boxplus t}: t \geq 1\right\}$ was that its Cauchy-Stieltjes transform satisfies an analytic subordination property in the sense of Littlewood: for each $t \geq 1$ there exists an analytic self-map $\omega_{t}$ of the complex upper half-plane so that $G_{\mu} \circ \omega_{t}=G_{\mu^{\boxplus t}}$, as shown in [5, 6, 9. We shall present our result in terms of analytic functions on $\{b \in \mathcal{B}: \Im b>0\}$ as in [20, 10, but it is fairly straightforward to see that all functions involved have fully matricial extension in the sense of [21].

Theorem 8.4. For any $\mu \in \Sigma^{0}(\mathcal{B})$ and $\alpha \in \mathcal{C P}(\mathcal{B})$ so that $\alpha-1$ is still completely positive there exists an analytic function $\omega_{\alpha}:\{b \in \mathcal{B}: \Im b>0\} \rightarrow\{b \in \mathcal{B}: \Im b>0\}$ so that $G_{\mu} \circ \omega_{\alpha}=G_{\mu} \boxplus \alpha$. The function $\omega_{\alpha}$ satisfies the functional equation

$$
\omega_{\alpha}(b)=b+(\alpha-1) h_{\mu}\left(\omega_{\alpha}(b)\right), \quad \Im b>0 .
$$

Proof. Let $f:\{b \in \mathcal{B}: \Im b>0\} \times\{b \in \mathcal{B}: \Im b>0\} \rightarrow\{b \in \mathcal{B}: \Im b>0\}$ be given by $f(b, w)=b+(\alpha-1) h_{\mu}(w)$. As shown in [7, Remark 2.5], $\Im h_{\mu}(w) \geq 0$ whenever $\Im w>0$. Moreover, as it is known from [13. that $h_{\mu}(w)=\sigma\left[(\mathcal{X}-b)^{-1}\right]-\mu[\mathcal{X}]$ for a completely positive map $\sigma$ of norm equal to the variance of $\mu$, on the set $\{w \in \mathcal{B}: \Im w>\Im b / 2\}, h_{\mu}$ is uniformly bounded by $M_{b}=2\|\mu[\mathcal{X}]\|+4\|\alpha\|_{\text {cp }} \cdot \| \mu[\mathcal{X}$. $\mathcal{X}]-\mu[\mathcal{X}] \cdot \mu[\mathcal{X}]\left\|_{\text {cp }} \cdot\right\|[\Im b]^{-1} \|+2$. Thus, the map $f(b, \cdot)$ maps the set $\{w \in \mathcal{B}: \Im w \geq$ $\left.\Im b / 2,\|w\| \leq 2 M_{b}\right\}$ inside its interior, and so, by [10, Theorem 3.1], there exists a unique fixed point of this map in the interior of this set. Thus, $\omega_{\alpha}$ is indeed well defined. Moreover,

$$
\omega_{\alpha}(b)=\lim _{n \rightarrow \infty} \underbrace{f(b, f(b, \cdots f}_{n \text { times }}(b, w) \cdots))
$$

for any $w \in M_{b}$, $\Im b>0$, which means that $\omega_{\alpha}$ is locally the uniform limit of a sequence of maps which are analytic in $b$, and hence it is analytic itself. 
Now, (8.9) is equivalent to the equation $\alpha \omega_{\alpha}(b)+(1-\alpha) G_{\mu}\left(\omega_{\alpha}(b)\right)^{-1}=b$, $\Im b>0$. If $G_{\mu} \circ \omega_{\alpha}=G_{\mu}{ }_{\alpha}$ indeed holds, then we must be able to verify the relation $\omega_{\alpha}\left(b^{-1}+\alpha R_{\mu}(b)\right)=b^{-1}+R_{\mu}(b)$; replacing in the above form of (8.9) gives $\alpha\left(b^{-1}+R_{\mu}(b)\right)+(1-\alpha) G_{\mu}\left(b^{-1}+R_{\mu}(b)\right)=b^{-1}+\alpha R_{\mu}(b)$, a relation trivially true from the definition of the $R$-transform. All these formulas hold for $b$ invertible of small enough norm. Analytic continuation allows us to conclude the proof.

While, as in 6], we have proved in the above proposition the existence of the subordination function without any recourse to any other tool except for analytic function theory, unlike in [6], we are not able to conclude from the above the existence of $\mu^{\boxplus \alpha}$. The missing ingredient is a good characterization of maps on the operatorial upper half-plane which are operator-valued Cauchy-Stieltjes transforms.

\section{EXAMPLES}

Example 9.1. For $\lambda \in \mathcal{B}$ symmetric, we define $\delta_{\lambda} \in \Sigma^{0}(\mathcal{B})$ by

$$
\delta_{\lambda}\left[\mathcal{X} b_{1} \mathcal{X} \ldots \mathcal{X} b_{n}\right]=\lambda b_{1} \lambda \ldots \lambda b_{n}
$$

or more generally $\mu[P]=P(\lambda)$. Then

$$
\left(\delta_{\lambda}\right)^{\boxplus \alpha}=\left(\delta_{\lambda}\right)^{\uplus \alpha}=\delta_{\alpha[\lambda]} .
$$

Example 9.2. If $\gamma_{\eta}$ is a centered $\mathcal{B}$-valued semicircular distribution, then

$$
R_{\gamma_{\eta}}\left[\mathcal{X} b_{1} \mathcal{X} b_{2} \ldots b_{n-1} \mathcal{X}\right]=\delta_{n, 2} \eta\left[b_{1}\right] .
$$

So

$$
\gamma_{\eta}=\left(\gamma_{I}\right)^{\boxplus \eta} .
$$

In forthcoming work we will describe $\mathcal{B}$-valued free Meixner distributions, which include the examples above as well as many others.

The following definition generalizes Definition 4.4.1 of [16].

Definition 9.3. Let $\nu \in \Sigma(\mathcal{B})$, and $\alpha \in \mathcal{C P}(\mathcal{B})$. The generalized free compound Poisson distribution $\pi_{\nu, \alpha}^{\boxplus} \in \Sigma(\mathcal{B})$ is determined by

$$
R_{\pi_{\nu, \alpha}^{\boxplus}}=(1-\alpha) \circ \delta_{0}+\alpha \circ \nu
$$

or more precisely by

$$
R_{\pi_{\nu, \alpha}^{\boxplus}}^{[n]}\left(b_{1}, b_{2}, \ldots, b_{n-1}\right)=\alpha\left[M_{\nu}^{[n]}\left(b_{1}, b_{2}, \ldots, b_{n-1}\right)\right] .
$$

In particular, $\pi_{\nu, \alpha}^{\boxplus}=\left(\pi_{\nu, I}^{\boxplus}\right)^{\boxplus \alpha}$. Similarly, generalized Boolean compound Poisson distributions are determined by

$$
B_{\pi_{\nu, \alpha}^{\uplus}}^{[n]}\left(b_{1}, b_{2}, \ldots, b_{n-1}\right)=\alpha\left[M_{\nu}^{[n]}\left(b_{1}, b_{2}, \ldots, b_{n-1}\right)\right] .
$$

Example 9.4. Let $\mathcal{B}=M_{2}(\mathbb{C}), \nu=\delta_{1}$, and

$$
\alpha[b]=\left(\begin{array}{ll}
0 & 1 \\
1 & 0
\end{array}\right) b\left(\begin{array}{ll}
0 & 1 \\
1 & 0
\end{array}\right) .
$$

Suppose $\pi_{\nu, \alpha}^{\boxplus}=\pi_{\mu, t}^{\boxplus}$ for some $\mu \in \Sigma(\mathcal{B})$ and $t>0$. Then

$$
\begin{aligned}
M_{\mu}^{[n]}\left(b_{1}, b_{2}, \ldots, b_{n-1}\right) & =\frac{1}{t} R_{\pi_{\nu, \alpha}^{\boxplus}}^{[n]}\left(b_{1}, b_{2}, \ldots, b_{n-1}\right) \\
& =\frac{1}{t} \alpha\left[M_{\nu}^{[n]}\left(b_{1}, b_{2}, \ldots, b_{n-1}\right)\right]=\frac{1}{t} \alpha\left[b_{1} b_{2} \ldots b_{n-1}\right] .
\end{aligned}
$$


In particular,

$\mu\left[(1-b \mathcal{X})^{*}(1-b \mathcal{X})\right]=1-b \mu[\mathcal{X}]-\mu[\mathcal{X}] b^{*}+\mu\left[\mathcal{X} b^{*} b \mathcal{X}\right]=1-\frac{1}{t}\left(b \alpha[1]+\alpha[1] b^{*}\right)+\frac{1}{t} \alpha\left[b^{*} b\right]$.

So for $\alpha$ as above and $b=\left(\begin{array}{cc}t & 0 \\ 0 & 0\end{array}\right)$,

$$
\mu\left[(1-b \mathcal{X})^{*}(1-b \mathcal{X})\right]=\left(\begin{array}{cc}
1-2 & 0 \\
0 & 1+t
\end{array}\right)
$$

is not positive. It follows that such $\mu, t$ do not exist, and so the class of distributions in the preceding definition is wider than Definition 4.4.1 of [16].

We end the paper with an alternative operator model for Boolean compound Poisson distributions.

Example 9.5. Let $\mu$ be a Boolean compound Poisson distribution. Also, let $\alpha$ : $\mathcal{B} \rightarrow \mathcal{B}$ be a completely positive map which has the special form

$$
\alpha[b]=(1+e) b\left(1+e^{*}\right)
$$

for some $e \in \mathcal{B}$.

We can choose a noncommutative probability space $\left(\mathcal{M}=\mathcal{B} \oplus \mathcal{M}_{0}, \mathbb{E}, \mathcal{B}\right)$ and a selfadjoint noncommutative random variable $Y \in \mathcal{M}_{0}$ satisfying

$$
M_{Y}=B_{\mu}
$$

By taking a further Boolean product (with amalgamation over $\mathcal{B}$ ) we may assume that $\mathcal{M}_{0}$ contains an element $Q$ which is Boolean independent from $Y$ over $\mathcal{B}$ and satisfies

$$
\mathbb{E}(Q)=e, \quad \operatorname{Var}_{Q}(b)=b,
$$

where

$$
\operatorname{Var}_{Q}(b)=\mathbb{E}\left(\left(1+Q^{*}\right) b(1+Q)\right)-\mathbb{E}\left(1+Q^{*}\right) b \mathbb{E}(1+Q) .
$$

Theorem 9.6. In the setting of the preceding example, let

$$
T=(1+Q) Y\left(1+Q^{*}\right)
$$

Then the distribution of $T$ is $\mu^{\uplus \alpha}$.

Proof. By Boolean independence of $Y$ and $Q$,

$$
\begin{aligned}
M_{T}^{[1]} & =\mathbb{E}(T) \\
& =(1+\mathbb{E}(Q)) \mathbb{E}(Y)\left(1+\mathbb{E}(Q)^{*}\right) \\
& =(1+\mathbb{E}(Q)) M_{Y}^{[1]}\left(1+\mathbb{E}(Q)^{*}\right),
\end{aligned}
$$

so $M_{T}^{[1]}=\alpha\left(M_{Y}^{[1]}\right)$. 
To compute $M_{T}^{[n]}(b, \ldots, b)$ for $n \geq 2$ and $b \in \mathcal{B}$, we set

$$
R_{i}:=\left(1+Q^{*}\right) b_{i}(1+Q)-b_{i}=Q^{*} b_{i}+b_{i} Q+Q^{*} b_{i} Q .
$$

Note that $R_{i} \in \mathcal{M}_{0}$. Then, since $R_{i}$ is Boolean independent from $Y$ over $\mathcal{B}$, one has:

$$
\begin{aligned}
& M_{T}^{[n]}\left(b_{1}, b_{2}, \ldots, b_{n-1}\right) \\
& =\mathbb{E}\left(T b_{1} T b_{2} \cdots T b_{n-1} T\right) \\
& =\mathbb{E}\left((1+Q) Y\left(1+Q^{*}\right) b_{1}(1+Q) \cdots Y\left(1+Q^{*}\right) b_{n-1}(1+Q) Y\left(1+Q^{*}\right)\right) \\
& =\mathbb{E}\left((1+Q) Y\left(b_{1}+R_{1}\right) Y \cdots\left(b_{n-1}+R_{n-1}\right) Y\left(1+Q^{*}\right)\right) \\
& =\sum_{1 \leq m_{1}<\ldots<m_{p}=n} \mathbb{E}\left((1+Q)\left(Y b_{1} \ldots b_{m_{1}-1} Y\right) R_{m_{1}}\right. \\
& \left.\cdots R_{m_{1}+\ldots+m_{p-1}}\left(Y b_{m_{1}+\ldots+m_{p-1}+1} \ldots b_{n-1} Y\right)\left(1+Q^{*}\right)\right) \\
& =\sum_{1 \leq m_{1}<\ldots<m_{p}=n} \mathbb{E}(1+Q) \mathbb{E}\left(Y b_{1} \ldots b_{m_{1}-1} Y\right) \mathbb{E}\left(R_{m_{1}}\right) \\
& \cdots \mathbb{E}\left(R_{m_{1}+\ldots+m_{p-1}}\right) \mathbb{E}\left(Y b_{m_{1}+\ldots+m_{p-1}+1} \ldots b_{n-1} Y\right) \mathbb{E}\left(1+Q^{*}\right) \\
& =\sum_{1 \leq m_{1}<\ldots<m_{p}=n} \mathbb{E}(1+Q) M_{Y}^{\left[m_{1}\right]}\left(b_{1}, \ldots, b_{m_{1}-1}\right) \mathbb{E}\left(R_{m_{1}}\right) \\
& \cdots \mathbb{E}\left(R_{m_{1}+\ldots+m_{p-1}}\right) M_{Y}^{\left[m_{p}\right]}\left(b_{m_{1}+\ldots+m_{p-1}+1}, \ldots, b_{n-1}\right) \mathbb{E}\left(1+Q^{*}\right) .
\end{aligned}
$$

Now using

$$
\begin{aligned}
\mathbb{E}\left(R_{i}\right) & =\mathbb{E}\left(\left(1+Q^{*}\right) b_{i}(1+Q)\right)-b_{i} \\
& =\operatorname{Var}_{Q}\left(b_{i}\right)+\mathbb{E}\left(1+Q^{*}\right) b_{i} \mathbb{E}(1+Q)-b_{i}=\mathbb{E}\left(1+Q^{*}\right) b_{i} \mathbb{E}(1+Q),
\end{aligned}
$$

we get

$$
\begin{aligned}
M_{T}^{[n]}\left(b_{1}, b_{2}, \ldots, b_{n-1}\right) & \\
= & \sum_{1 \leq m_{1}<\ldots<m_{p}=n}\left(\mathbb{E}(1+Q) M_{Y}^{\left[m_{1}\right]}\left(b_{1}, \ldots, b_{m_{1}-1}\right) \mathbb{E}\left(1+Q^{*}\right)\right) b_{m_{1}} \\
& \quad \ldots b_{m_{1}+\ldots+m_{p-1}}\left(\mathbb{E}(1+Q) M_{Y}^{\left[m_{p}\right]}\left(b_{m_{1}+\ldots+m_{p-1}+1}, \ldots, b_{n-1}\right) \mathbb{E}\left(1+Q^{*}\right)\right) .
\end{aligned}
$$

Using Möbius inversion and formula (4.6), it follows that

$$
\begin{aligned}
B_{T}^{[n]}\left(b_{1}, b_{2}, \ldots, b_{n-1}\right) & =\mathbb{E}(1+Q) M_{Y}^{[n]}\left(b_{1}, \ldots, b_{n-1}\right) \mathbb{E}\left(1+Q^{*}\right) \\
& =(1+e) M_{Y}^{[n]}\left(b_{1}, \ldots, b_{n-1}\right)\left(1+e^{*}\right) \\
& =(1+e) B_{\mu}^{[n]}\left(b_{1}, \ldots, b_{n-1}\right)\left(1+e^{*}\right) \\
& =\alpha\left(B_{\mu}^{[n]}\left(b_{1}, \ldots, b_{n-1}\right)\right) .
\end{aligned}
$$

This proves that the distribution of $T$ is $\mu^{\uplus \alpha}$.

Added in proof: An alternative proof of Theorem[7.9 was found by Dimitri Shlyakhtenko in "On operator-valued free convolution powers", arXiv:1110.5127 [math.OA], 2011. 


\section{ACKNOWLEDGMENT}

This work was started when the authors participated in a Research in Teams project (10rit159) at the Banff International Research Station, in August 2010. The support of BIRS and its very inspiring environment are gratefully acknowledged.

\section{REFERENCES}

[1] Michael Anshelevich, Free evolution on algebras with two states, J. Reine Angew. Math. 638 (2010), 75-101. MR2595336

[2] Serban T. Belinschi and Alexandru Nica, $\eta$-series and a Boolean Bercovici-Pata bijection for bounded k-tuples, Adv. Math. 217 (2008), no. 1, 1-41. MR2357321 MR2357321(2009c:46088)

[3] _ On a remarkable semigroup of homomorphisms with respect to free multiplicative convolution, Indiana University Math. J. 57 (2008), no. 4, 1679-1713. MR2440877 (2009f:46087)

[4] , Free Brownian motion and evolution towards $\boxplus$-infinite divisibility for $k$-tuples, Internat. J. Math. 20 (2009), no. 3, 309-338. MR2500073 MR2500073 (2010g:46108)

[5] Serban T. Belinschi and Hari Bercovici, Atoms and regularity for measures in a partially defined free convolution semigroup, Math. Z., 248, (2004), no. 4, 665-674. MR2103535 (2006i:46095)

[6] , Partially Defined Semigroups Relative to Multiplicative Free Convolution, Internat. Math. Res. Not., 2005, no.2, 65-101. MR2128863 (2006f:46061)

[7] Serban T. Belinschi, Mihai Popa, and Victor Vinnikov, Infinite divisibility and a noncommutative Boolean-to-free Bercovici-Pata bijection, J. Funct. Anal. 262, (2012), no. 1, 94-123. MR 2852257

[8] Hari Bercovici and Vittorino Pata, Stable laws and domains of attraction in free probability theory, Ann. of Math. (2) 149 (1999), no. 3, 1023-1060, With an appendix by Philippe Biane. MR.1709310 (2000i:46061)

[9] Stephen Curran, Analytic subordination for free compression, preprint arXiv:0803.4227v2 [math.0A], 2008.

[10] J. William Helton, Reza Rashidi Far, and Roland Speicher, Operator-valued semicircular elements: solving a quadratic matrix equation with positivity constraints, Int. Math. Res. Not. IMRN (2007), no. 22, Art. ID rnm086, 15. MR2376207 (2008k:15017)

[11] Alexandru Nica and Roland Speicher, On the multiplication of free $N$-tuples of noncommutative random variables, Amer. J. Math. 118 (1996), no. 4, 799-837. MR1400060 (98i:46069)

[12] Alexandru Nica and Roland Speicher, Lectures on the combinatorics of free probability, London Mathematical Society Lecture Note Series, vol. 335, Cambridge University Press, Cambridge, 2006. MR2266879 (2008k:46198)

[13] Mihai Popa and Victor Vinnikov, Non-commutative functions and non-commutative free Levy-Hincin formula, arXiv:1007.1932v2 [math.0A], 2010.

[14] Dimitri Shlyakhtenko, Random Gaussian band matrices and freeness with amalgamation, Internat. Math. Res. Notices 1996, no. 20, 1013-1025. MR1422374 (97j:46070)

[15] _ A-valued semicircular systems, J. Funct. Anal. 166 (1999), no. 1, 1-47. MR.1704661 (2000j:46124)

[16] Roland Speicher, Combinatorial theory of the free product with amalgamation and operatorvalued free probability theory, Mem. Amer. Math. Soc. 132 (1998), no. 627, x+88. MR.1407898 (98i:46071)

[17] Roland Speicher and Reza Woroudi, Boolean convolution, Free probability theory (Waterloo, ON, 1995), Fields Inst. Commun., vol. 12, Amer. Math. Soc., Providence, RI, 1997, pp. 267279. MR 1426845 (98b:46084)

[18] Dan Voiculescu, Symmetries of some reduced free product $C^{*}$-algebras, Operator algebras and their connections with topology and ergodic theory (Buşteni, 1983), Lecture Notes in Math., vol. 1132, Springer, Berlin, 1985, pp. 556-588. MR799593 (87d:46075)

[19] _ Addition of certain noncommuting random variables, J. Funct. Anal. 66 (1986), no. 3, 323-346. MR839105 (87j:46122)

[20] - Operations on certain non-commutative operator-valued random variables, Astérisque (1995), no. 232, 243-275, Recent advances in operator algebras (Orléans, 1992). MR 1372537 (97b:46081) 
[21] The coalgebra of the free difference quotient and free probability, Internat. Math. Res. Notices 2000, no. 2, 79-106. MR1744647 (2001d:46096)

Department of Mathematics, Texas A\&M University, College Station, Texas 778433368

E-mail address: manshel@math.tamu.edu

Department of Mathematics and Statistics, University of Saskatchewan, 106 Wiggins Road, Saskatoon, Saskatchewan, Canada S7N 5E6 - And - Institute of Mathematics "Simion Stollow" of the Romanian Academy, Bucharest, Romania

E-mail address: belinsch@math.usask.ca

Institut de Mathématiques de Toulouse, Equipe de Statistique et Probabilités, F31062 Toulouse Cedex 09, France

E-mail address: fevrier@math.univ-toulouse.fr

Current address: Laboratoire de Mathématiques, Université Paris Sud, Bât. 425, 91405 Orsay Cedex, France

E-mail address: maxime.fevrier@math.u-psud.fr

Department of Pure Mathematics, University of Waterloo, Waterloo, Ontario, CANAdA N2L 3G1

E-mail address: anica@math.uwaterloo.ca 